\title{
A combined statistical bias correction and stochastic downscaling method for precipitation
}

\author{
Claudia Volosciuk ${ }^{1}$, Douglas Maraun ${ }^{2}$, Mathieu Vrac ${ }^{3}$, and Martin Widmann ${ }^{4}$ \\ ${ }^{1}$ GEOMAR Helmholtz Centre for Ocean Research Kiel, Kiel, Germany \\ ${ }^{2}$ Wegener Center for Climate and Global Change, University of Graz, Graz, Austria \\ ${ }^{3}$ Laboratoire des Sciences du Climat et de l'Environnement (LSCE), CNRS/IPSL, Gif-sur-Yvette, France \\ ${ }^{4}$ School of Geography, Earth and Environmental Sciences, University of Birmingham, Birmingham, UK \\ Correspondence to: Claudia Volosciuk (cvolosciuk@geomar.de)
}

Received: 5 August 2016 - Discussion started: 15 September 2016

Revised: 3 March 2017 - Accepted: 6 March 2017 - Published: 22 March 2017

\begin{abstract}
Much of our knowledge about future changes in precipitation relies on global (GCMs) and/or regional climate models (RCMs) that have resolutions which are much coarser than typical spatial scales of precipitation, particularly extremes. The major problems with these projections are both climate model biases and the gap between gridbox and point scale. Wong et al. (2014) developed a model to jointly bias correct and downscale precipitation at daily scales. This approach, however, relied on pairwise correspondence between predictor and predictand for calibration, and, thus, on nudged simulations which are rarely available. Here we present an extension of this approach that separates the downscaling from the bias correction and in principle is applicable to free-running GCMs/RCMs. In a first step, we bias correct RCM-simulated precipitation against gridded observations at the same scale using a parametric quantile mapping $\left(\mathrm{QM}_{\text {grid }}\right)$ approach. In a second step, we bridge the scale gap: we predict local variance employing a regression-based model with coarse-scale precipitation as a predictor. The regression model is calibrated between gridded and point-scale (station) observations. For this concept we present one specific implementation, although the optimal model may differ for each studied location. To correct the whole distribution including extreme tails we apply a mixture distribution of a gamma distribution for the precipitation mass and a generalized Pareto distribution for the extreme tail in the first step. For the second step a vector generalized linear gamma model is employed. For evaluation we adopt the perfect predictor experimental setup of VALUE. We also compare our method to the classical QM as it is usu-
\end{abstract}

ally applied, i.e., between $\mathrm{RCM}$ and point scale $\left(\mathrm{QM}_{\mathrm{point}}\right)$. Precipitation is in most cases improved by (parts of) our method across different European climates. The method generally performs better in summer than in winter and in winter best in the Mediterranean region, with a mild winter climate, and worst for continental winter climate in Mid- and eastern Europe or Scandinavia. While $\mathrm{QM}_{\text {point }}$ performs similarly (better for continental winter) to our combined method in reducing the bias and representing heavy precipitation, it is not capable of correctly modeling point-scale spatial dependence of summer precipitation. A strength of this two-step method is that the best combination of bias correction and downscaling methods can be selected. This implies that the concept can be extended to a wide range of method combinations.

\section{Introduction}

To assess the impacts of hydrometeorological extremes in a changing climate, high-quality precipitation projections on the point scale are often demanded. Much of our knowledge about future changes in precipitation is based on global (GCMs) and/or regional climate models (RCMs). These have resolutions which are much coarser than typical spatial scales of processes relevant for precipitation. This concerns particularly extreme precipitation, which is far more sensitive to resolution than mean precipitation (Volosciuk et al., 2015). Although horizontal resolution of GCMs has successively increased since the first assessment 
report of the Intergovernmental Panel on Climate Change (IPCC, 1990), resolving all important spatial and temporal scales remains beyond current computational capabilities for transient global climate change simulations (Le Treut et al., 2007). The simulation of precipitation depends heavily on processes that are parameterized in current GCMs, and also in most RCMs (Flato et al., 2013). Biases related to parameterization schemes and unresolved processes thus remain in addition to systematic biases related to the large-scale circulation (e.g., Flato et al., 2013; Kotlarski et al., 2014).

Different approaches have been employed to downscale and/or reduce biases of simulated precipitation, particularly extremes: (a) high-resolution GCMs, (b) dynamical downscaling using RCMs that are nested in the GCMs (Rummukainen, 2010), and (c) statistical downscaling including post-processing with bias-correction methods (Maraun et al., 2010). But even though high-resolution GCMs and RCMs improve the representation of extreme precipitation by better resolving mesoscale atmospheric processes, biases remain and there is still a scale gap between the simulated gridbox values of precipitation and point-scale data (i.e., rain gauges). Hence, statistical bias-correction methods are also applied to such high-resolution simulations. These so-called model output statistics (MOS) approaches employ a correction function derived in present-day simulations to future simulations of the same model (Maraun et al., 2010).

Quantile mapping (Piani et al., 2009), one example MOS approach, is widely applied to statistically post-process simulated precipitation. While this might be a reasonable approach for correcting biases on the same spatial scale, variability on local scales is not fully determined by grid-scale variability, e.g., the exact location, size, or intensity of a thunderstorm. This is part of the representativeness problem between gridbox and point values (Zwiers et al., 2013). Quantile mapping is a deterministic approach that cannot add random variability. It simply inflates the variance leading to an overestimation of spatial extremes, and too smooth a variance in space and also in time (von Storch, 1999; Maraun, 2013a). Gridbox precipitation, e.g., is the area average of sub-grid precipitation. The aggregation averages local variations in time such that gridbox time series are smoother in time than local time series. Quantile mapping can not overcome this mismatch in temporal structure (apart from correcting the drizzle effect). This temporal effect is more difficult to trace than the spatial effect (Maraun, 2013a). Standard downscaling approaches in turn have a limited ability to correct systematic biases. Wong et al. (2014) developed a model that jointly bias corrects and downscales precipitation at daily scales. However, this approach relies on pairwise correspondence between predictor and predictand for calibration that is only provided by nudged GCM/RCM simulations, and is not able to post-process standard, free-running GCM simulations (Eden et al., 2014).

Here we present a modification of the Wong et al. (2014) approach that is designed to also work in princi- ple for free-running GCM/RCMs, such as those available from ENSEMBLES (van der Linden and Mitchell, 2009) or CORDEX (e.g., Jacob et al., 2013). With the aim of combining their respective advantages we combine a statistical bias correction and a stochastic downscaling method. Thereby we separate bias correction from downscaling by inserting a gridded observational dataset as a reference between these two steps. In particular, as a first step we apply a parametric quantile mapping approach between an RCM and a gridded observational dataset. In a second step we bridge the scale gap between gridded and point scale by employing a stochastic regression-based model that is calibrated between gridded and station observations and then applied to the biascorrected precipitation from the first step.

In Sect. 2 the general concept is introduced; the data used are described in Sect. 3. In Sect. 4 we present the bias correction and the stochastic downscaling model. Results of the evaluation of our model for example stations across Europe are provided in Sect. 5 and, finally, Sect. 6 contains the conclusion.

\section{General concept}

We separate bias correction from downscaling into two steps to overcome the shortcomings of each method and to combine their respective strengths. Our concept is illustrated schematically in Fig. 1. In the first step, we use the advantage of distribution-wise bias correction (i.e., the correction function is calibrated on long-term distributions) to eliminate systematic biases in the RCM. While this distribution-wise setting may correct systematic RCM biases, it cannot bridge the gap between gridbox and point scale for two reasons. First, a considerable portion of subgrid variability is random for precipitation and has to be modeled as stochastic noise. However, distribution-wise MOS methods are deterministic and do thus not add unexplained random variability. Second, distribution-wise methods cannot separate local variability into systematic explained variability and small-scale unexplained variability. Moreover, when simulated shortterm variability is inflated to match local variability, longterm trends are also inflated (Maraun, 2013a). Therefore, we only apply this distribution-wise method to correct biases on the same spatial scale; i.e., as a reference we use gridded observations on the same grid as the RCM. In the second step, we employ a stochastic regression-based model to overcome the representativeness problem. This regression model corrects systematic local effects (e.g., whether a rain gauge is positioned on the lee or windward side of a mountain). It also adds random (unexplained) small-scale variability, in contrast to approaches of combined methods that employ spatial interpolation for downscaling (Wood and Maurer, 2002; Wood et al., 2004; Payne et al., 2004) or rescale the grid-scale precipitation with a factor to match the observations (Ahmed et al., 2013). We calibrate the probabilistic regression model 


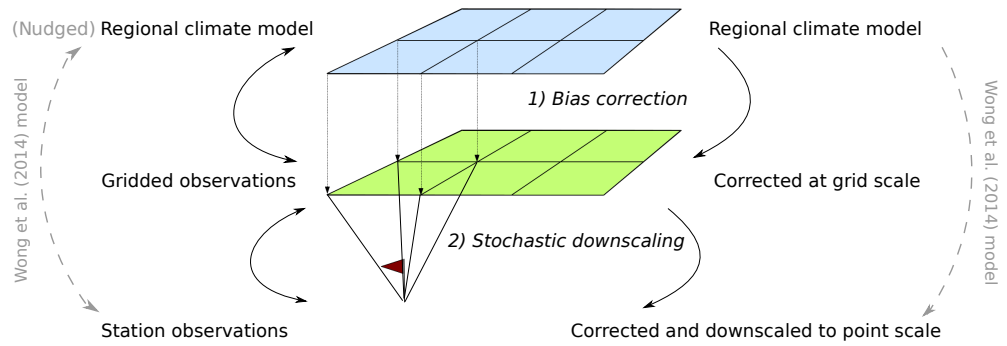

Figure 1. Schematic of (black) our combined statistical bias correction and stochastic downscaling model, and (grey) the Wong et al. (2014) model.

between gridded and point-scale observations and then apply it to the corrected grid-scale time series in the validation period. This corresponds to a perfect prog (PP) setting for the regression, while the bias is corrected in the first step. This combined approach is an extension of the model by Wong et al. (2014) that jointly bias corrects and downscales precipitation (see Fig. 1). They employ a probabilistic regression model that is calibrated between RCM and point-scale observations (MOS approach). It requires nudged RCM simulations for calibration since temporal correspondence is essential.

With this concept in place, basically in the first step any reasonable distribution-wise MOS approach, and in the second step any adequate stochastic model, can be employed. A strength of this concept is its flexibility; i.e., the best suitable combination of statistical models for a given location and season can be determined. In this study, we employ a quantile mapping $\left(\mathrm{QM}_{\text {grid }}\right)$ approach based on the mixture distribution of a gamma and generalized Pareto distribution (Vrac and Naveau, 2007) in the first step. The model used in the second step consists of a logistic regression for wet day probabilities and a vector generalized linear model predicting the parameters of a gamma probability distribution (VGLM gamma) for precipitation intensities. Note that this combination of methods may not be optimal in all studied locations. However, the aim of this study is to introduce and evaluate the concept of this combined approach rather than to find the optimal specific implementation for all studied locations.

To evaluate and illustrate our method, we adopt the perfect predictor experimental setup of the VALUE framework (Maraun et al., 2015). Employing the same evaluation framework as VALUE allows for comparison of our method to all models participating in the VALUE experiment. In this context, a reanalysis-driven RCM is used which allows us to evaluate the ability of the method to correct RCM biases, before evaluating GCM-driven simulations where biases of both GCMs and RCMs need to be corrected. We note that although this is a pairwise setup where simulated and observed weather states are in principle synchronized (with the exception of the internal variability generated within the RCM), we only use the simulated and observed distributions for the bias correction. Thus, as explained above, the approach can be transferred to any simulation setup, e.g., GCM-driven RCM simulations or GCM simulations. For comparison we also applied the classical QM approach, i.e., directly between RCM and point scale $\left(\mathrm{QM}_{\text {point }}\right)$.

The method is evaluated by 5 -fold cross-validation for the time period 1979-2008; i.e., five 6-year long periods are predicted by the model that was fitted to the remaining 24 years. Artificial predictive skill is thus not present as the predicted period is not part of the training period. The model is fitted and evaluated for each season separately; 86 stations across Europe are studied (as selected for the VALUE experiment; see Fig. 2) representing different climates. In the evaluation of our model we compare eight European subdomains (dashed lines in Fig. 2): the British Isles (BI), the Iberian Peninsula (IP), France (FR), Mid-Europe (ME), Scandinavia (SC), the Alps (AL), the Mediterranean (MD), and eastern Europe (EA). These domains have been defined within the PRUDENCE project (Christensen and Christensen, 2007) and are often used for RCM evaluation (e.g., Kotlarski et al., 2014). Although climatic differences within these subdomains remain, they summarize European climate zones and intercomparison amongst them allows for study of large-scale gradients (e.g., from maritime (west) to continental (east) or from cold (north) to mild (south) winters). We slightly extended the PRUDENCE regions SC, AL, and MD such that all studied rain gauges are included in the analysis.

\section{Data and gridbox selection}

As prescribed by the perfect predictor experiment within the VALUE framework, we use the RACMO2 RCM from the KNMI (van Meijgaard et al., 2012) to test our method for the time period from 1979 to 2008 . The RCM has been driven with ERA-Interim reanalysis (Dee et al., 2011) within the EURO-CORDEX framework (Jacob et al., 2013). The simulation has been carried out at a horizontal resolution of $0.44^{\circ}$ $(\sim 50 \mathrm{~km})$ over a rotated grid. Note that the resolution we em- 


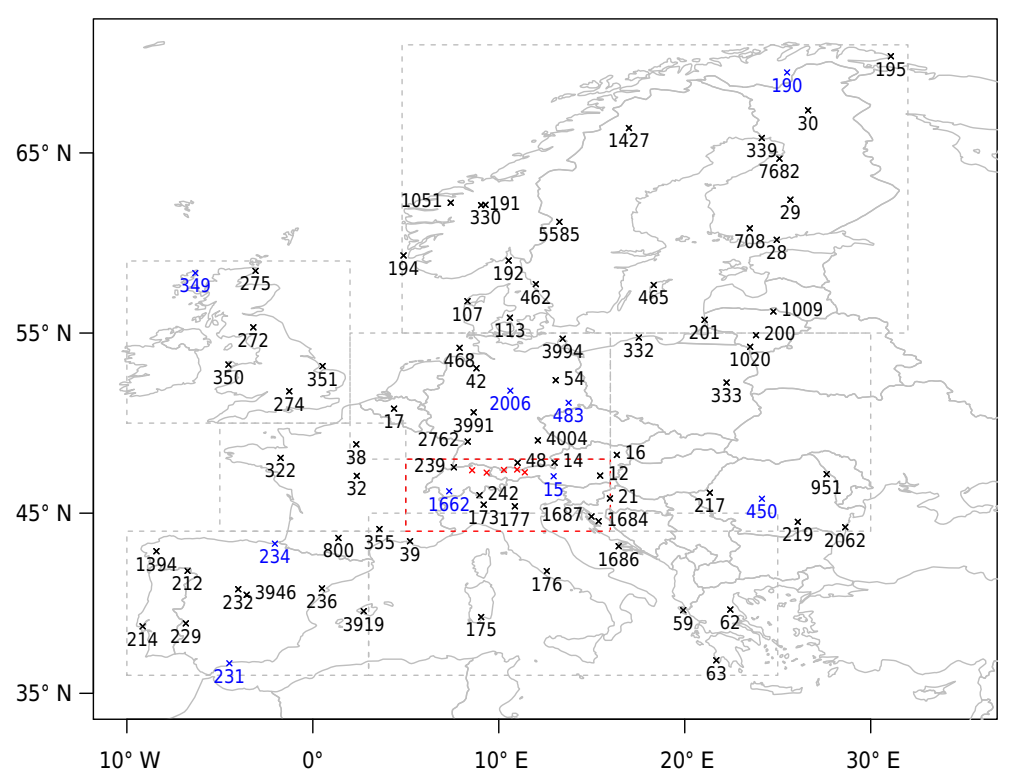

Figure 2. Location and IDs of used rain gauges from ECA\&D. IDs of red marked stations from left to right: 244, 243, 4002, 58, and 13. Stations for detailed analysis are marked in blue. Dashed lines represent European subdomains for analysis as defined by the PRUDENCE project (Christensen and Christensen, 2007): the British Isles (BI), the Iberian Peninsula (IP), France (FR), Mid-Europe (ME), Scandinavia (SC), the Alps (AL, dashed red line), the Mediterranean (MD), and eastern Europe (EA).

ploy $\left(0.44^{\circ}\right)$ differs from the resolution used in the VALUE experiment $\left(0.11^{\circ}\right)$.

As the gridded observational dataset, E-OBS version 10 (Haylock et al., 2008) is used, also at $0.44^{\circ}$ resolution. The reason for choosing the $0.44^{\circ}$ horizontal resolution for both $\mathrm{RCM}$ and E-OBS is that the actual resolution of E-OBS might in some regions be lower than the nominal $0.22^{\circ}$ due to sparse rain gauge density included in the dataset ${ }^{1}$. Gridding very few rain gauges to a high resolution might in particular result in overly smooth extremes (Haylock et al., 2008; Hofstra et al., 2009a, b; Maraun et al., 2011a). Hence, too high a resolution of a gridded dataset may be an unreliable reference for bias correction, at least for summer extreme events. Moreover, this could cause artificial smoothing of extremes by bias correction. In some regions where station density is very sparse, this might even hold true for the chosen resolution. Although E-OBS is probably not an appropriate reference in some regions, it is the best available gridded dataset covering the whole EURO-CORDEX domain.

The E-OBS reference gridbox for both steps (bias correction and downscaling) is generally the closest gridbox to the respective station. If the closest gridbox is an ocean gridbox (i.e., for coastal and island stations) and only contains missing values, we select the gridbox with the highest correlation in winter between daily precipitation at the given station and the five closest E-OBS gridboxes. In winter the spatial decorrelation length of precipitation is generally large, implying

\footnotetext{
${ }^{1}$ For station density of actual E-OBS versions, refer to the ECA\&D website: http://www.ecad.eu/dailydata/datadictionary.php.
}

that several gridboxes are often affected by the same weather system, and, thus, the gridbox with the most similar climate can be reliably identified.

The RCM gridbox that is bias-corrected and downscaled is generally chosen as the closest gridbox to the E-OBS reference gridbox - also for coastal and island stations where the chosen RCM gridbox might thus differ from the closest RCM gridbox to the final reference (i.e., rain gauge). For locations in the rain shadows we choose the RCM gridbox which best represents the climate at the given location to correct overly low precipitation values caused by not enough windward air masses crossing the mountain range ("location bias", Maraun and Widmann, 2015). To this end, the highest correlation between the winter seasonal mean of RCM and gridded observations within $250 \mathrm{~km}$ around the closest gridbox to the observations is determined. Note that when transferring this approach to free-running RCM simulations this gridbox selection step needs to be carried out employing a reanalysis-driven simulation of the same RCM to ensure temporal correspondence.

For local-scale observations we used 86 stations across Europe from ECA\&D (Klein Tank et al., 2002) selected by the VALUE experimental framework (Maraun et al., 2015). The locations and ids of these stations are illustrated in Fig. 2. A detailed analysis is carried out for some example stations representing different climates (highlighted in blue in Fig. 2). 


\section{Statistical model}

\subsection{Step 1: bias correction}

In our model we correct several biases. In a first step, the "location bias" is corrected by gridbox selection (see Sect. 3 for details). In the second step, the "drizzle" effect is corrected by increasing the wet day threshold for the RCM such that the number of wet days (closely) matches the gridded observations, with a threshold of $0.1 \mathrm{~mm} \mathrm{day}^{-1}$. Finally, we correct precipitation intensities of wet days (i.e., exceeding the corrected wet day threshold) using a quantile mapping $(\mathrm{QM})$ approach which is described in the following. The correction function $y=f(x)$ between the simulated $(x)$ and corrected $(y)$ values of daily precipitation intensities such that the corrected values match the observations is based on the cumulative distribution functions (cdfs) as $\operatorname{cdf}_{\text {obs }}(f(x))=\operatorname{cdf}_{\mathrm{RCM}}(x)$ (Piani et al., 2009). To allow for extrapolation in a future climate to unobserved precipitation intensities and to avoid deterioration of future extremes that might occur with an approach that relies on empirical cdfs, we chose a parametric QM approach.

To model precipitation intensities the gamma distribution is commonly used (Katz, 1977). While the bulk of precipitation is generally well represented, the tail of the gamma distribution is usually too light to capture high and extreme rainfall intensities (e.g., Vrac and Naveau, 2007; Maraun et al., 2010). Thus, an extreme value distribution, such as the generalized Pareto (GP) distribution (Coles, 2001), might be required to model the extremes of the precipitation distribution. To correct the whole precipitation distribution, including extreme tails, we apply the mixture distribution of Vrac and Naveau (2007) which consists of a gamma distribution for the precipitation mass and a GP distribution for the extreme tail. This model is a variant of Frigessi et al. (2002). The distribution $l_{\phi}(x)$ of observed precipitation $x$ on wet days is modeled as

$l_{\phi}(x)=c(\boldsymbol{\phi})\left(\left\{\left[1-w_{m, \tau}(x)\right] f_{\lambda, \gamma}(x)\right\}+\left[w_{m, \tau}(x) g_{\xi, \sigma}(x)\right]\right)$,

$\boldsymbol{\phi}=(\lambda, \gamma, \xi, \sigma, m, \tau)$,

where $f_{\lambda, \gamma}$ is the probability density function (pdf) of the gamma distribution with the rate parameter $\lambda$ and the shape parameter $\gamma$,

$f_{\lambda, \gamma}(x)=\frac{\lambda^{\gamma}}{\Gamma(\gamma)} x^{\gamma-1} e^{-\lambda x}, \quad \lambda, \gamma>0$,

and $g_{\xi, \sigma}$ is the pdf of the GP distribution:

$g_{\xi, \sigma}(x)=\frac{1}{\sigma}\left[1+\frac{\xi(x-u)}{\sigma}\right]^{-\frac{1}{\xi}-1}$ for $x \geq u$,

with the scale parameter $\sigma>0$ and the shape parameter $\xi$ which determines the tail behavior of the GP distribution as follows: $\xi<0$ : bounded tail; $\xi \rightarrow 0$ : exponential distribution (light tailed); and $\xi>0$ : infinite heavy tail. Here, we constrain $\xi \geq 0$ to ensure that our model can be applied to a future climate that may experience higher values than those observed during the present-day training period for the model. The function $w_{m, \tau}$ is a weight function that determines the transition between the gamma and GP pdfs as

$w_{m, \tau}(x)=\frac{1}{2}+\frac{1}{\pi} \arctan \left(\frac{x-m}{\tau}\right), \quad m, \tau>0$,

with the location parameter $m$ denoting the location of the center of this transition and the transition rate $\tau$ influencing the rapidity of the transition between the two distributions. To finally obtain the mixture pdf, the mixture function (Eq. 1) must be normalized, which is carried out here by multiplying the mixture function by a constant $c(\phi)$. In the mixture pdf (Eq. 1) the threshold $u$ in the GP distribution (Eq. 3) is set to zero, as the location parameter $m$ of the weight function (Eq. 4) fulfills the purpose of a threshold in Eq. (1). Moreover, setting the threshold to zero and applying a weight function instead also solves the problem of threshold selection with unsupervised estimation and avoids discontinuity in the mixture pdf $l_{\phi}(x)$ (Eq. 1) (Vrac and Naveau, 2007). The parameters for $l_{\phi}(x)$ are estimated using maximum likelihood estimation (MLE). For technical details on the implementation of this model, please refer to Appendix A1.

Since the mixture model is a complex model with six free parameters, a thorough statistical model selection is necessary. We select between the mixture model and the simpler gamma-only model separately for the observed $\left(F_{\text {obs }}\right)$ and RCM-simulated $\left(F_{\mathrm{RCM}}\right)$ distributions. For the selection, we apply the Akaike information criterion (AIC, Akaike, 1973), which asymptotically selects the model that minimizes the mean squared error between prediction and observation (Shao, 1997). The AIC is defined as $-2 \log (L)+2 k$ with the likelihood $L$ corresponding to the maximum likelihood estimate of the $k$ model parameters. The AIC is dominated by the most densely populated region of the distribution. Hence, a good fit for the bulk of the distribution (and thus a low AIC) might nevertheless come with large biases in the extremes (see Appendix A2 for an example). To avoid a model choice with unreasonably high extremes, we therefore introduce a criterion based on a comparison between the 100 season return levels estimated by the mixture model (Eq. 1) and by the GP distribution (Eq. 3) before the AIC-based model selection is applied. For technical details on these model selection procedures, please refer to Appendix A2.

To strictly avoid bias correction deteriorating the predictor and introducing biases, both the complete cross-validated corrected time series and the raw RCM output are compared to gridded observations as a reference using the Cramér-von Mises (CvM) criterion. The CvM is a measure of the distance between two empirical cdfs (cdf bias hereafter; Darling, 1957) and has been used to evaluate cdf-based correction models before (e.g., Michelangeli et al., 2009; Vrac 
et al., 2012). If $\operatorname{cdf}_{\mathrm{ref}}(x)$ is the empirical cdf of observations as a reference (i.e., the perfect bias correction would match this reference) and $\operatorname{cdf}_{\text {corr }}(x)$ is the empirical cdf of the biascorrected time series, the CvM statistics is defined as the integrated squared difference between $\mathrm{cdf}_{\text {ref }}$ and $\mathrm{cdf}_{\text {corr }}$ as follows:

$\mathrm{CvM}=\int_{-\infty}^{\infty}\left|\operatorname{cdf}_{\text {corr }}(x)-\operatorname{cdf}_{\text {ref }}(x)\right|^{2} \mathrm{~d} x$.

Here, the CvM is computed for both the corrected daily precipitation time series and the uncorrected RCM-simulated precipitation time series with E-OBS as a reference. The predictor for downscaling is selected based on the lower CvM. In other words, the bias-corrected time series is only used as a predictor for the downscaling step if it improves the predictor compared to the raw uncorrected RCM.

\subsection{Step 2: stochastic downscaling}

To bridge the scale gap we apply the regression model developed by Wong et al. (2014) as follows. We determine the statistical relationship between gridded and station observations. This statistical relationship is then applied to coarsescale precipitation as a predictor which is selected in the

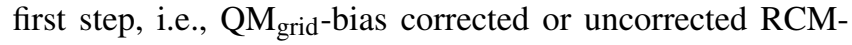
simulated precipitation. To be able to estimate the distribution of precipitation as a function of a given predictor, a stationary distribution is not sufficient. The family of generalized linear models (GLMs) extends linear regression to such purposes (e.g., Dobson, 2001). In this framework the timedependent expectation of a random variable is linked via a monotonic link function to a linear combination of predictors. The logistic regression model belongs to the class of GLMs and is often used to model the changing probability of rainfall occurrence (Chandler and Wheater, 2002). We model the probability $p_{i}$ of a day $i$ being wet (i.e., greater than the threshold selected earlier at $0.1 \mathrm{~mm} \mathrm{day}^{-1}$ ) as a function of coarse-scale precipitation $x_{i}$ as

$h\left(p_{i}\right)=\log \left(\frac{p_{i}}{1-p_{i}}\right)=\alpha x_{i}+\beta$,

where $h(\cdot)$ is the logit link function and the parameters $\alpha$ and $\beta$ are estimated by MLE. The logit link function gives the logarithm of the odds.

Subsequently, precipitation intensity on wet days is modeled using a vector generalized linear model (VGLM) as a regression model (Yee and Wild, 1996; Yee and Stephenson, 2007). VGLMs are an extension of GLMs. While GLMs describe the conditional mean of a wide range of distributions, VGLMs allow for prediction of a vector of parameters from the same set of predictors, which is useful if one is also interested in the variance or the extremes of a distribution. Wong et al. (2014) implemented a mixture model version (see Eq. 1) and a gamma model version (see Eq. 2) employing a VGLM. Here we apply the VGLM gamma version since the calibration and model selection procedure for the VGLM mixture model is computationally rather expensive. The simpler gamma model might be sufficient here as in the downscaling step a predictor is employed that already explains a large portion of the variance. The quality of downscaled precipitation does not only depend on the chosen model, but also on the quality of the predictor. Employing the mixture model for the bias-correction step is thus meaningful to ensure a good representation of higher quantiles and extremes in the predictor, although downscaling is performed with a simpler gamma model. The scale $\theta$ (the inverse of $\lambda$ in Eq. 2) and the shape $\gamma$ parameters depend linearly on the predictor (coarse-scale precipitation) $x_{i}$. The model has the form

$\theta_{i}=\theta_{0}+\psi_{\theta} x_{i}$

$\gamma_{i}=\gamma_{0}+\psi_{\gamma} x_{i}$

where the regression parameters $\psi_{\theta}$, and $\psi_{\gamma}$ are estimated by MLE.

Combining the probability of wet day occurrence and the gamma model distribution defining the precipitation intensities, we get the probability that observed precipitation on a given day $\left(R_{i}\right)$ is less than or equal to a particular precipitation intensity $(r)$ :

$\operatorname{Pr}_{\theta, \gamma}\left(R_{i} \leq r\right)=\Gamma_{\theta, \gamma}\left(R_{i} \leq r \mid W\right) p_{i}+\left(1-p_{i}\right)$,

where $\Gamma_{\theta, \gamma}\left(R_{i} \leq r \mid W\right)$ is the gamma cdf and $p_{i}$ is the probability of that given day being wet.

\subsection{Evaluation metrics}

We evaluate our combined model based on the following metrics.

- Mean bias: absolute difference between seasonal means as (model - reference).

- cdf bias: CvM criterion which represents the mean squared error of a cdf compared to a reference cdf (for details see Sect. 4.1).

- \%sim > perc $95_{\text {obs }}$ : percentage of simulated wet days exceeding the observed 95 th percentile.

- QQ plots: the quantiles (i.e., sorted time series) of modeled precipitation are plotted against the quantiles of the reference. For the evaluation of the second step (downscaling) standardized QQ plots are used which are explained in Sect. 5.2.2.

- Spatial autocorrelation: correlation of a variable with itself in geographical space. The correlogram is estimated by centered Mantel statistics using R package ncf (Bjornstad, 2015). The correlation for a set of distances 

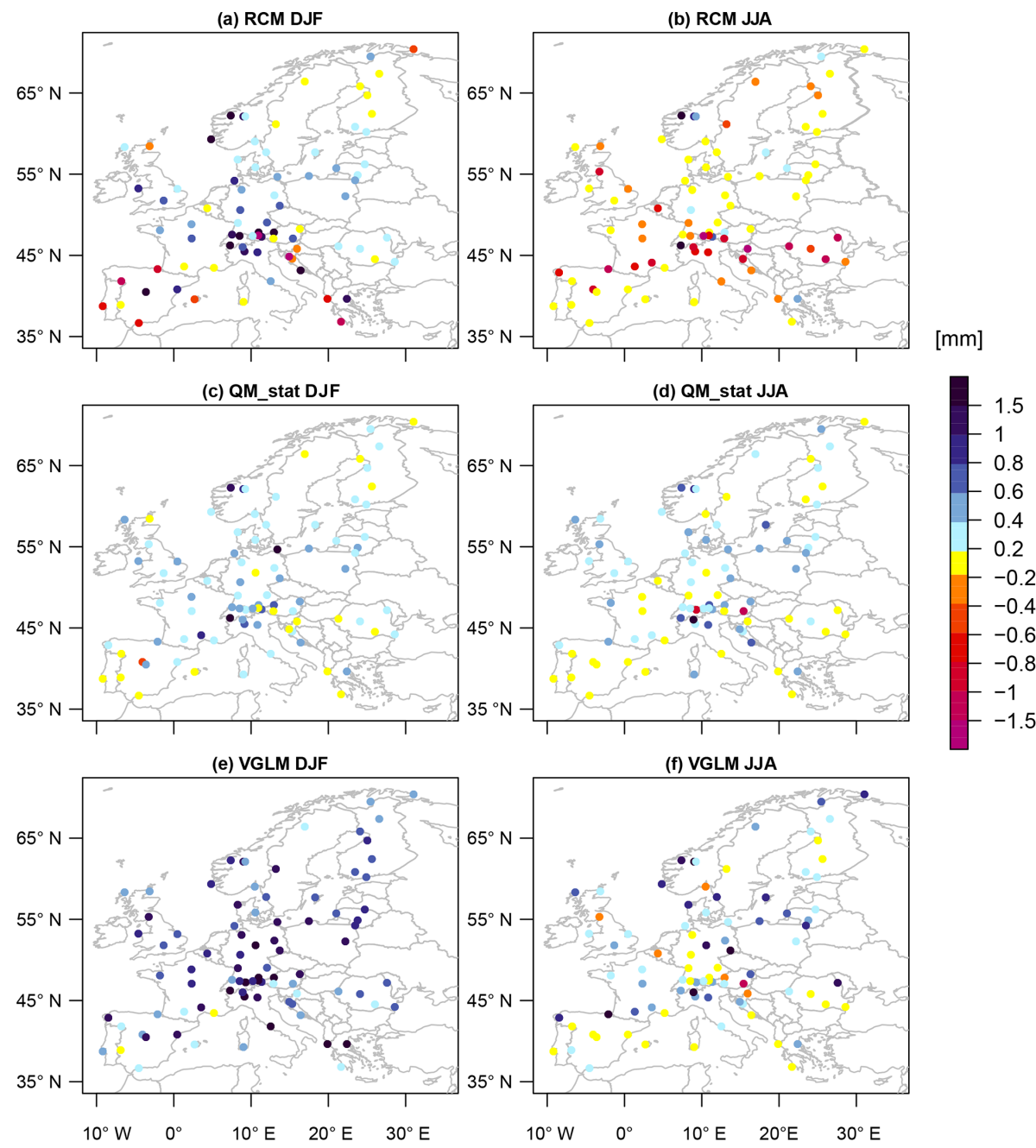

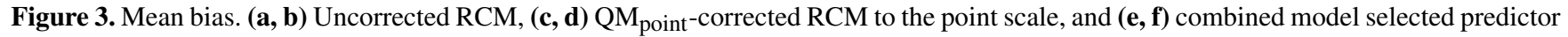
(RCM or $\mathrm{QM}_{\text {grid }}$-corrected RCM) and VGLM. Reference is station data.

at discrete distance classes is calculated. Significance is assessed by 1000 random permutations. The correlogram is estimated for daily values and then averaged. For the VGLM the correlogram is computed for 100 realizations of the stochastic model and then averaged. The correlogram is centered on zero; i.e., zero represents similarity across the region. Crossing the zero line implies thus that the pair of distances is not more similar than what would be expected by chance alone across the region.

\section{Results}

We first evaluate the mean bias of our combined model (selected predictor and VGLM) against station observations and compare it to the raw uncorrected RCM and to classical $\mathrm{QM}_{\text {point }}$ (between RCM and point scale). Then the performance of the two steps (bias correction and downscaling) is assessed individually and in combination. Finally, all analyzed models are compared. The evaluation is carried out for the time period 1979-2008 by analyzing the cross-validated (5-fold) time series. The first step (bias correction) is evaluated against the gridded E-OBS dataset, although E-OBS might underrepresent the extremes in some regions where station density is sparse. The second step (downscaling) and the combined model (steps 1 and 2) are evaluated against station observations.

\subsection{Evaluation of mean precipitation bias}

Figure 3 shows the mean bias of precipitation (against station observations) as modeled by (a, b) the RCM and (c, d) the classical $\mathrm{QM}_{\text {point }}$ approach applied directly between RCM and station observations and (e, f) our combined model. The RCM has a stronger bias in DJF than in JJA. In DJF it is rather too wet, whereas in JJA many locations have a dry 

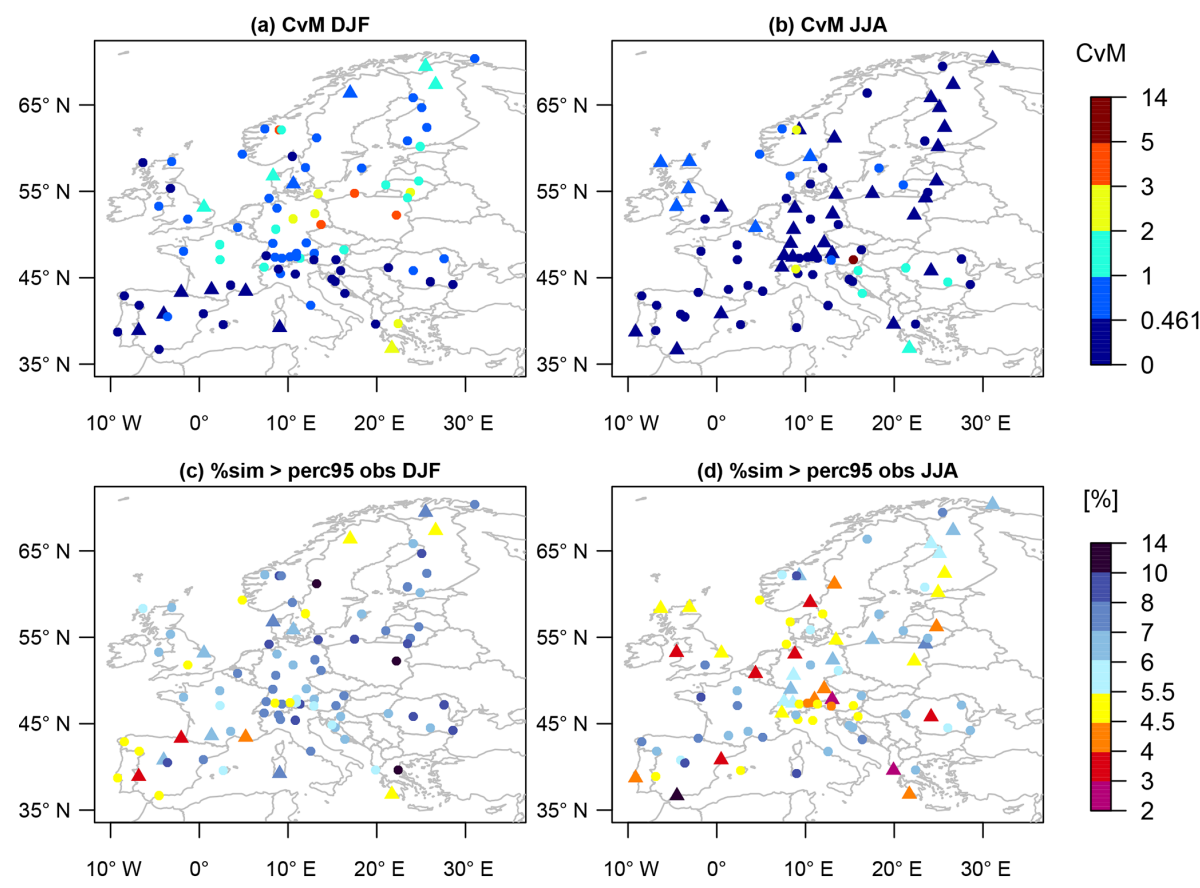

[\%]

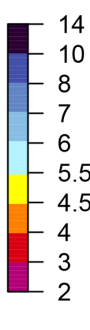

Figure 4. Step 1: bias correction to grid scale. (a, b) CvM score for the selected cross-validated predictor against E-OBS. Threshold for values under which the model cdf is not statistically significantly different at the $95 \%$ level from the reference cdf: 0.461 . (c, d) Percentage of wet days in the CvM-selected cross-validated predictor exceeding the 95 th percentile of wet days in E-OBS $\left(\%\right.$ sim $>$ perc $\left.95_{\text {obs }}\right)$. Selected

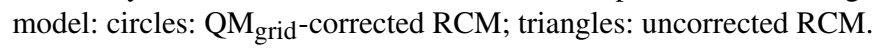

bias. In both seasons the bias is improved by $\mathrm{QM}_{\text {point }}$, with a slight remaining wet bias. Our combined model also improves the mean bias of the RCM in JJA. However, in DJF wet biases remain and got even worse in some locations. This raises the question why the results become worse when statistical post-processing is applied. However, the bias of the seasonal mean does not give information on how the precipitation distribution is represented or the predictive power of the model. These issues are evaluated in the following.

\subsection{Evaluation of the combined model}

First, both steps of the combined model are evaluated individually. Second, the combination of both steps is evaluated. In this combined model the predictor selected in the first step is used for the regression model in the second step.

\subsubsection{Evaluation of Step 1: bias correction vs. E-OBS}

Figure 4 shows the cross-validated selected predictor (uncorrected RCM: triangles; $\mathrm{QM}_{\text {grid-corrected RCM: circles) that }}$ is used in the second step for downscaling. For predictor selection we apply the Cramér-von Mises score (CvM, Eq. 5, Sect. 4.1) which represents the mean squared error of a cdf compared to a reference cdf (cdf bias hereafter). The predictor is selected based on the lowest CvM score of the crossvalidated $\mathrm{QM}_{\text {grid-corrected time series and the raw uncor- }}$ rected RCM with gridded observations as a reference. Gen- erally our bias correction often improves precipitation. It is selected 73 times in December-February (DJF) and 49 times in June-August (JJA) out of 86 rain gauges.

The CvM values of the selected predictor (Fig. 4a and b) indicate that the cdf bias is generally lower in JJA than in DJF. In DJF the cdf bias is lowest in the Mediterranean region, with a mild winter climate. However, the CvM criterion is quite sensitive to small deviations between the cdfs. The highest selected CvM values are found for Graz (Austria) in JJA, and Leba (northern Poland), Siedlce (eastern Poland), and Dresden (eastern Germany) in DJF. QQ plots for these high CvM values (see Appendix B1) suggest that the corrected time series are still usable and show improvements compared to the raw RCM, although they are of course not a perfect match of the observations. These remaining inaccuracies of the $\mathrm{QM}_{\text {grid }}$ approach can be related to both a time-varying correction function and the parametric correction function. Figure 5 summarizes Fig. 4 over the European subdomains by boxplots. Spatial variability throughout the subdomain is quantified by CvM variability represented by the box. In DJF the boxplots confirm the lowest cdf bias in the Mediterranean region (MD and IP) that is already visible on the map (Fig. 4a). The highest median is in ME. However, although the median is slightly lower than in ME, spatial variability is largest in EA, extending to the highest $\mathrm{CvM}$ values. This indicates that there are problems with continental winter climate which persist after bias correction as 
(a) CvM DJF

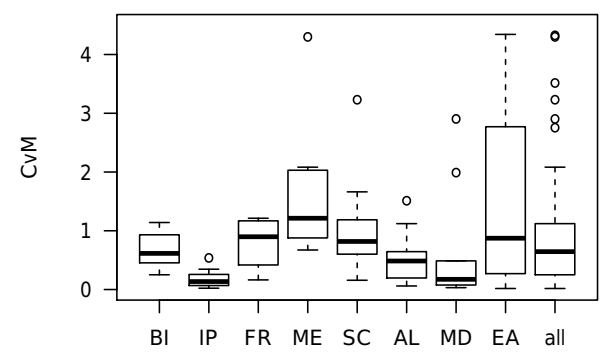

(c) \%sim > perc95 obs DJF (CvM sel)

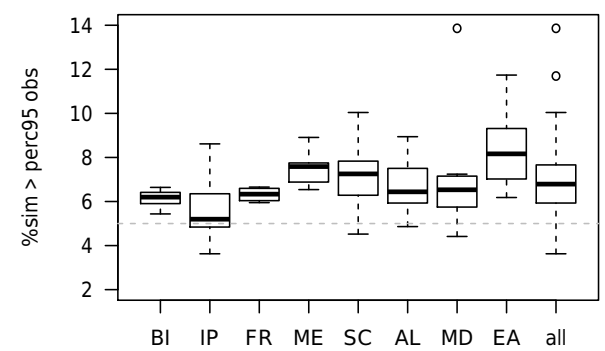

(b) CvM JJA

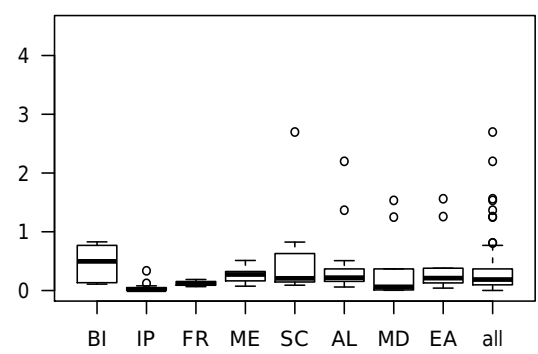

(d) \%sim > perc95 obs JJA (CvM sel)

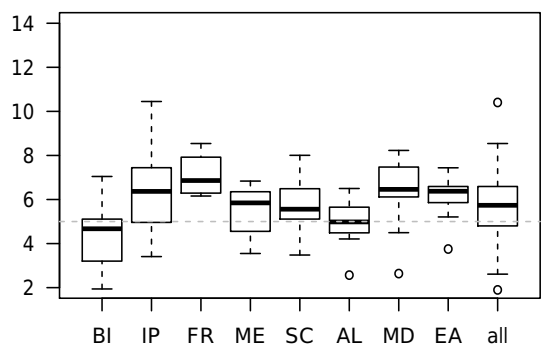

Figure 5. Step 1: bias correction to grid scale. Boxplots of (a, b) CvM score and (c, d) percentage of simulated wet days exceeding the observed 95th percentile (\%sim > perc95 ${ }_{\text {obs }}$ ) for the CvM-selected cross-validated predictor in European subdomains: British Isles (BI), Iberian Peninsula (IP), France (FR), Mid-Europe (ME), Scandinavia (SC), Alps (AL), Mediterranean (MD), and eastern Europe (EA). Outlier out of range in (b) AL and all: 12.95.

in ME and EA mostly the bias-corrected model is selected (Fig. 4a). QQ plots of the two worst examples in EA (Leba and Siedlce; Appendix B1) show that the complete precipitation time series remains too wet, whereas in the worst example of ME (Dresden; Appendix B1) the bias correction performs well for most values and only fails in the highest quantile. In JJA the CvM score, and hence the cdf bias, is very low, and no pronounced differences between the subdomains can be identified (Fig. 5a).

The representation of heavy precipitation by the selected predictor is evaluated by the percentage of simulated values that are higher than the 95th percentile of the observations on wet days (\%sim > perc $95_{\text {obs }}$, Figs. $4 \mathrm{c}, \mathrm{d}$ and $\left.5 \mathrm{c}, \mathrm{d}\right)$. Thus, in a "perfect" model this would be exactly $5 \%$ (yellow). In many locations there are slightly too many "extremes"; i.e., the occurrence of heavy precipitation ( $>$ perc $\left.95_{\text {obs }}\right)$ is overestimated, particularly in DJF. Consistent with the CvM score, the overestimation in heavy-precipitation occurrence increases in DJF from west to east (FR $\rightarrow \mathrm{ME} \rightarrow \mathrm{EA})$ and is again highest in EA, followed by ME and SC (Fig. 5c). In JJA the occurrence of heavy precipitation is quite well represented in AL and BI (Fig. 5d); it is, however, underestimated in some locations (Fig. 4d). In the other subregions the occurrence of heavy precipitation is also slightly overestimated in JJA (Fig. 5d).

\subsubsection{Evaluation of Step 2: downscaling vs. station}

Here we present some examples to illustrate the performance of the VGLM gamma for different climates, calibrated between gridded (E-OBS) and point-scale (station) observations. All results that are shown for the evaluation of the downscaling step (step 2, Figs. 6-7 and Appendix B2) are calibrated over the complete time period and then predicted by E-OBS as a predictor for the same time period. As we do not use the cross-validated time series here, the best possible relationship is presented. This allows us to evaluate the goodness-of-fit and is a necessary step before evaluating the model in a cross-validation setup. For a detailed evaluation of the VGLM gamma for the relationship between nudged RCM/GCM simulations and station observations over the British Isles, refer to Wong et al. (2014) and Eden et al. (2014).

To evaluate the goodness-of-fit, we use residual QQ plots (Fig. 6 for DJF and Appendix B2 for JJA). As a QQ plot requires quantiles of an unconditional distribution, we standardized the day-to-day varying distribution to a stationary gamma distribution $^{2}$ (Coles, 2001; Wong et al., 2014). This stationary distribution no longer has the predictor-dependent

\footnotetext{
${ }^{2}$ Standardization is performed as (1) compute probabilities for reference values (here: station observations) from an estimated nonstationary gamma distribution (i.e., gamma parameters depend on the predictor and, thus, vary from day to day); (2) compute quantiles of a gamma distribution with stationary parameters for these probabilities of a non-stationary distribution; (3) plot these quan-
} 

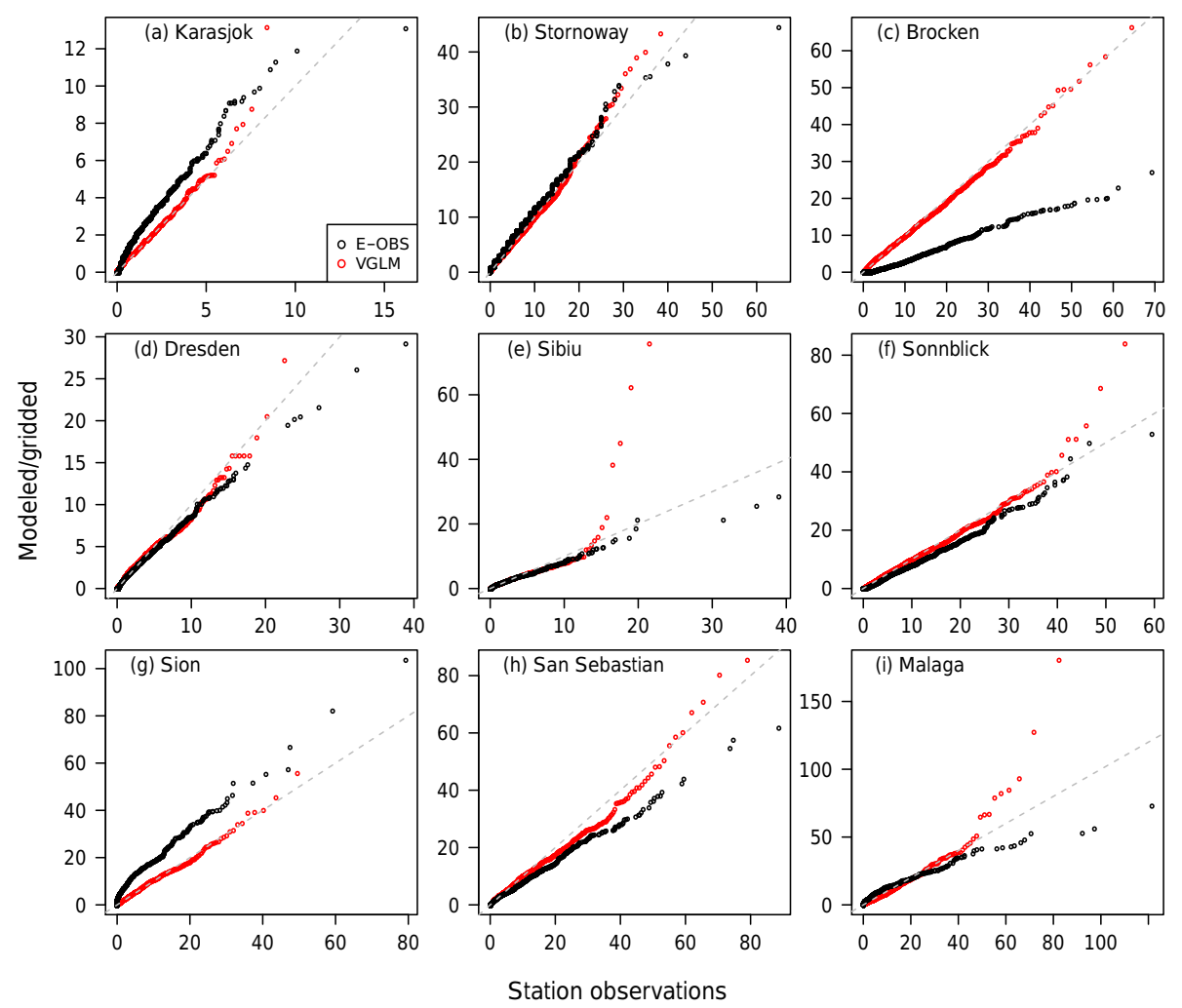

Figure 6. Step 2: downscaling. QQ plots for example stations in DJF. VGLM gamma standardized to the stationary gamma distribution fitted to observed wet day intensities between gridded and point-scale precipitation observations (mm day ${ }^{-1}$ ). (a) Karasjok, (b) Stornoway, (c) Brocken, (d) Dresden, (e) Sibiu, (f) Sonnblick, (g) Sion, (h) San Sebastian, and (i) Malaga.

day-to-day variations; i.e., the effect of the predictor is approximately removed. Due to this procedure the goodnessof-fit of the regression model can be evaluated separately, instead of evaluating only the combined effect of predictor and regression model which is present in the time-varying gamma parameters, and, thus, also in realizations drawn from these varying distributions. Therefore, deficiencies that are indicated by these standardized QQ plots are either due to inappropriate model structure or ill-fitting parameters. Note that the values of model and observation are shifted due to the standardization, depending on the strength of the predictor.

Improvements by the VGLM gamma compared to the predictor can be seen in most examples ranging from Scandinavia to the Mediterranean and from the Atlantic coast to eastern Europe in both seasons. However, in some locations the quantiles modeled by the VGLM gamma compare well to station observations (at least in Malaga, better than the predictor) up to a certain quantile (e.g., Sibiu, $\sim 12 \mathrm{~mm}$ day $^{-1}$, and Malaga, $\sim 42 \mathrm{~mm} \mathrm{day}^{-1}$, in DJF), while there is a wet bias for intensities of the higher quantiles. It has been verified that precipitation at these locations is gamma-distributed (not

tiles against quantiles of stationary gamma distribution for theoretical probabilities: $(1: n) /(n+1)$. shown). To understand this model behavior we analyze the predictor-predictand relationship of both observations and VGLM in Fig. 7 for DJF and Appendix B2 for JJA. Circles are the observed gridded against point-scale precipitation intensities, showing the spread of point-scale predictands for a given grid-scale predictor. The lines represent the 10, 25, 50 (median), 75, 90, and 95\% quantiles of the VGLM gamma model as a function of the predictor. This function of course fits best in the range where most of the values used to estimate the relationship are. For instance, in Sibiu (Malaga) for higher predictor values (Sibiu: $>15 \mathrm{~mm} \mathrm{day}^{-1}$; Malaga: $\left.>42 \mathrm{~mm} \mathrm{day}^{-1}\right)$ the predictands are around or below the $25 \%(50 \%)$ quantile of the model, and, thus, simulated systematically too high by the VGLM. In both cases the bulk of the distribution is well captured however. This problem is also visible at other stations, e.g., Dresden or Karasjok. In JJA it is even more pronounced (Appendix B2), particularly in Dresden and Sibiu, where the high predictor values are even below the modeled $10 \%$ quantile. These examples indicate that the VGLM basically allows for three different generalized linear relationships between the predictor and the parameters of the gamma distribution: concave (i.e., Brocken DJF), straight (i.e., San Sebastian DJF), or convex (i.e., Malaga DJF). No changes from lower to higher quan- 

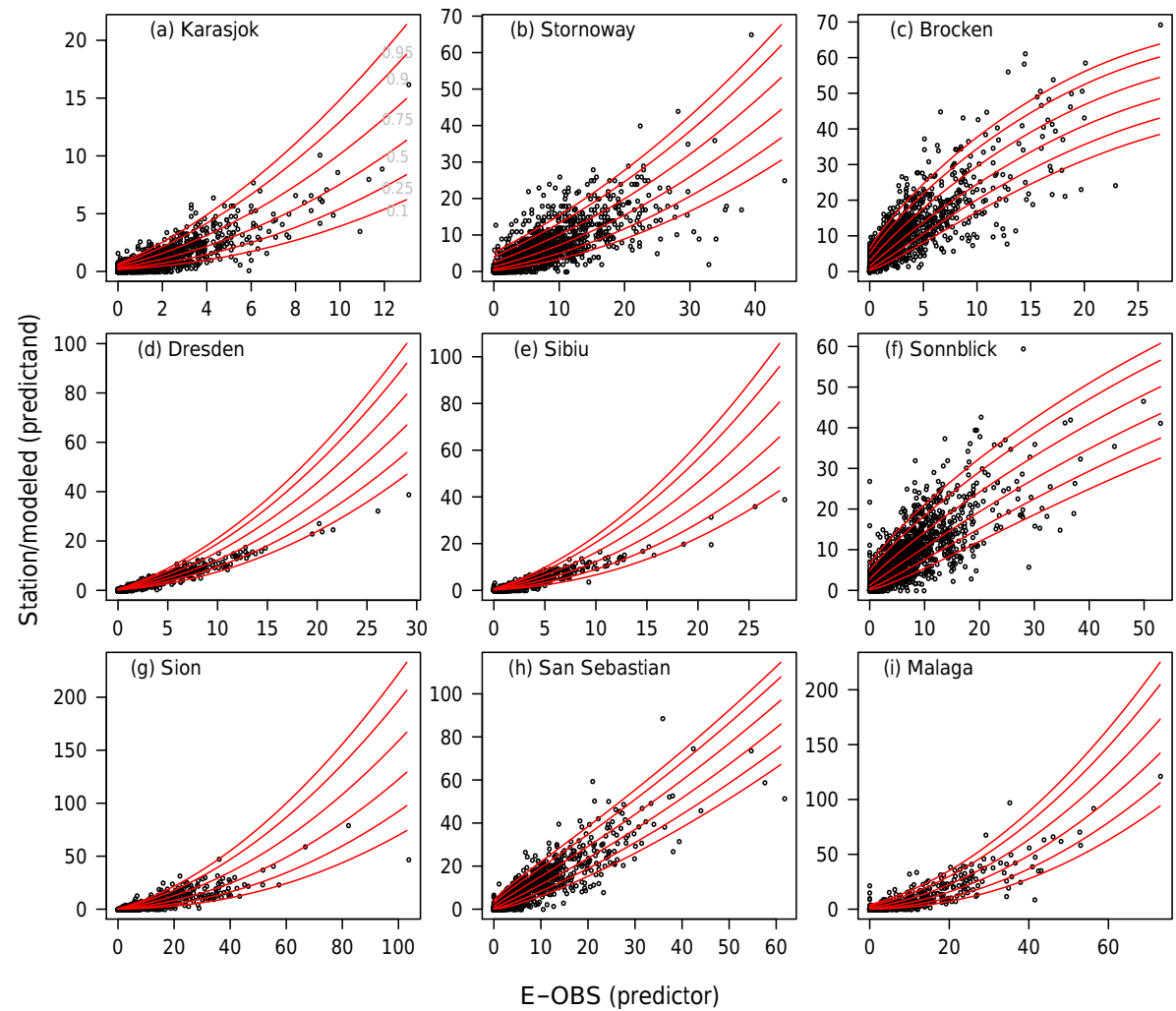

Figure 7. Step 2: downscaling. Estimated relation between gridded and point-scale precipitation observations for example stations in DJF. VGLM gamma where both parameters depend on the predictor fitted to observed wet day intensities. The predictor is E-OBS. Circles: observed precipitation intensities $\left(\mathrm{mm} \mathrm{day}^{-1}\right)$; lines: 0.1, 0.25, 0.5, 0.75, 0.9, and 0.95 modeled quantiles (mm day $\left.{ }^{-1}\right)$. (a) Karasjok, (b) Stornoway, (c) Brocken, (d) Dresden, (e) Sibiu, (f) Sonnblick, (g) Sion, (h) San Sebastian, and (i) Malaga.

tiles between these three types are possible. In some locations this appears to be not flexible enough to capture the true relationship, which can be nonlinear. A more flexible relationship that allows for a changed model behavior for higher values could improve the results but comes with the risk of overfitting. Additionally, in eastern Europe the station density included in E-OBS is low ${ }^{3}$. Hence, in the E-OBS gridbox closest to Sibiu, there may be only very few (one or two) stations included, implying most likely a misrepresentation of gridbox precipitation. This problem affects the calibration of the model where E-OBS is used as a reference as well as simulations employing E-OBS or precipitation that is corrected to E-OBS as a predictor. We do not show results of the crossvalidation here as the described problems with the VGLM in some locations are already present when repredicting the calibration period where the skill should be higher than in a cross-validation setup where a period is predicted that is not part of the calibration period. This clearly highlights deficiencies in the model for these locations.

In both DJF (Fig. 7) and JJA (Appendix B2) Sonnblick and Brocken show a concave function, whereas the function

\footnotetext{
${ }^{3}$ For station density of actual E-OBS versions refer to the ECA\&D website: http://www.ecad.eu/dailydata/datadictionary.php.
}

in the other example stations is generally convex. The rain gauges at Sonnblick and Brocken are on top of the respective mountain. Although their climate is quite different as Sonnblick is a high mountain in the Alps (altitude: $3106 \mathrm{~m}$ ), whereas the Brocken is the highest mountain in the northern German Harz low mountain range (altitude: $1142 \mathrm{~m}$ ), they have an exposed position, along with high variability, in common. These results show that the VGLM gamma is capable of modeling the scale relationship for such exposed places of high variability quite well.

\subsubsection{Evaluation of the combination of steps 1 and 2: bias correction and downscaling vs. station}

In the combined model the VGLM gamma, calibrated against E-OBS, is applied to the predictor selected in Sect. 5.2.1 (Fig. 4). Here we evaluate precipitation simulated by the combined model (predictor and VGLM) with station observations as a reference, and compare it to the uncorrected $\mathrm{RCM}$-simulated precipitation and to the $\mathrm{QM}_{\text {grid-corrected }}$ precipitation. The cross-validated time series are evaluated. For the VGLM the evaluation criteria were computed for 100 realizations and then averaged. 

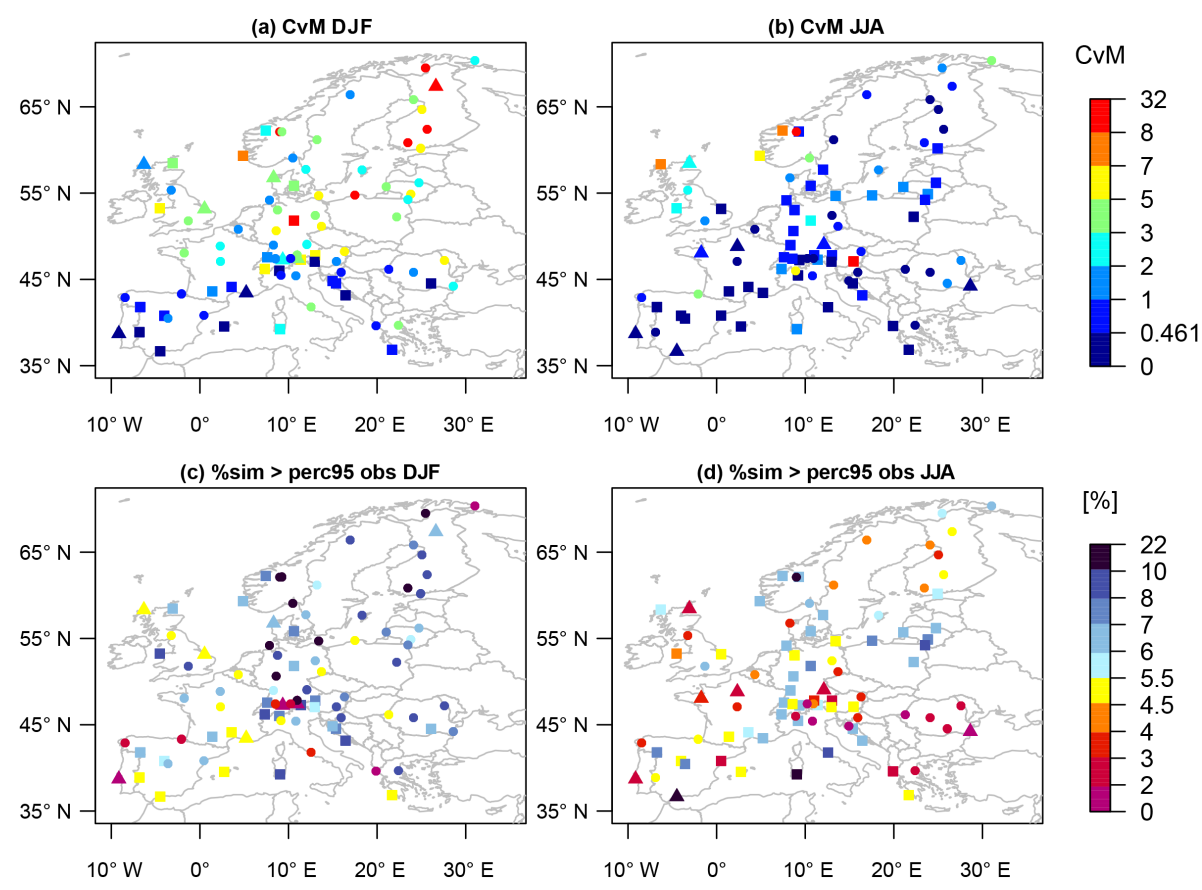

[\%]

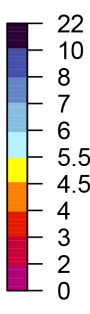

Figure 8. Steps 1 and 2: combined model. (a, b) CvM values for the selected cross-validated model. The threshold for values under which the model cdf is not statistically significantly different at the $95 \%$ level from reference cdf: 0.461 . (c, d) Percentage of cross-validated model values exceeding the 95 th percentile of station observations $\left(\%\right.$ sim $>$ perc $95_{\text {obs }}$ ) for the cross-validated CvM-selected model. For the VGLM the criteria were computed for 100 realizations and then averaged. Selected model: squares: combined model (predictor and VGLM); circles: $\mathrm{QM}_{\text {grid }}$-corrected RCM; triangles: uncorrected RCM. Note the different color scales than in Fig. 4.

To evaluate the predictor and VGLM combined model, we apply the same criteria as for the first step (bias correction, Sect. 5.2.1), but with station observations (i.e., point scale) as a reference. The CvM scores $(a, b)$ and the percentage of simulated values that are higher than the 95th percentile of the observations on wet days $\left(\% \operatorname{sim}>\right.$ perc $\left.95_{\text {obs }}, \mathrm{c}, \mathrm{d}\right)$ for the selected best model based on the CvM criterion are shown in Fig. 8 and summarized by boxplots for the European subdomains in Fig. 9. QQ plots for example stations are provided in Fig. 10 for DJF and in Appendix B3 for JJA. Precipitation is improved in most cases by (parts of) our method. The uncorrected RCM (Fig. 8, triangles) is only selected at eight (seven) stations in DJF (JJA). However, even if the RCM is selected, the other models do not necessarily perform much worse, such as in Stornoway in DJF (Fig. 10) or in Malaga in JJA (Appendix B3). The combined predictor and VGLM model (plotted as squares) is selected by CvM 25 times (45 times) in DJF (JJA). The more frequent selection of the VGLM in JJA compared to DJF is likely related to the dominant underlying mechanism; i.e., in summer there are many small-scale convective precipitation events, whereas in winter precipitation is mainly caused by largescale weather systems.

The CvM values of the selected model (Fig. 8a and b) indicate that the cdf bias is again generally lower in JJA than in DJF, and for DJF lowest in the Mediterranean region. In eastern Europe and Scandinavia in DJF the VGLM is only rarely selected - in these regions the $\mathrm{QM}_{\text {grid }}$-corrected time series which is on grid scale is mostly selected, although the reference cdf is on point scale (Fig. 8a). This might be due to problems with the VGLM gamma as explained in Sect. 5.2.2. The rather large cdf bias in ME, SC, and EA in DJF could hence be related to the remaining scale gap as the $\mathrm{QM}_{\text {grid }}{ }^{-}$ corrected time series is not expected to correctly represent the point scale. The QQ plot of Sibiu in DJF (Fig. 10) illustrates this problem. The higher $\mathrm{QM}_{\text {grid-corrected quantiles }}$ are as expected too low and the VGLM fails at this station in DJF (see also Sect. 5.2.2). Finding an adequate stochastic model to bridge the scale gap might improve the representation of precipitation in such cases. Also in JJA there are examples where the VGLM has not been selected, but a suitable VGLM would likely further improve the results (Appendix B3, San Sebastian, Dresden, and Karasjok). For, e.g., Brocken JJA and Sion DJF an improved VGLM may likely even improve the result although the VGLM has been selected. However, finding the optimal model for all 86 stations is beyond the scope of our study. The boxplots confirm again the good performance for DJF in the Mediterranean region (MD and IP), and also in AL (Fig. 9a). The CvM score and, thus, the cdf bias, are again very low in JJA, indicating good performance of our method with no pronounced difference between the European subregions (Fig. 9b). However, 
(a) CvM DJF

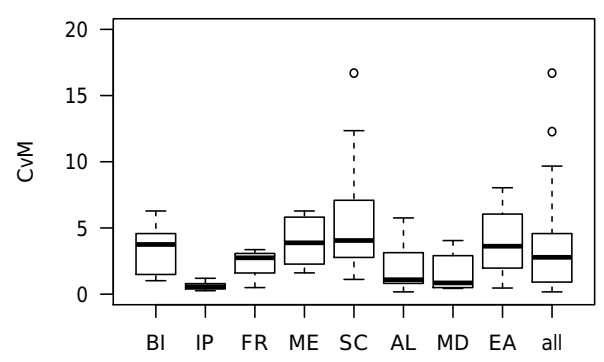

(c) \%sim > perc95 obs DJF (CvM sel)

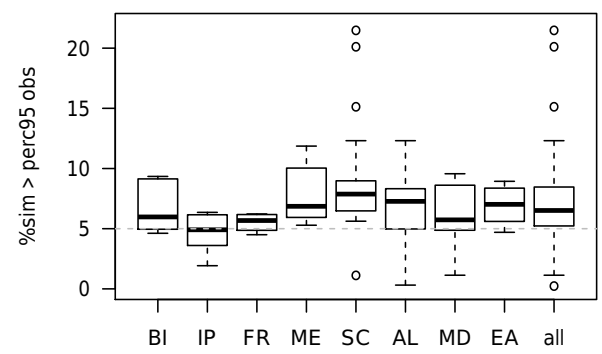

(b) CvM JJA

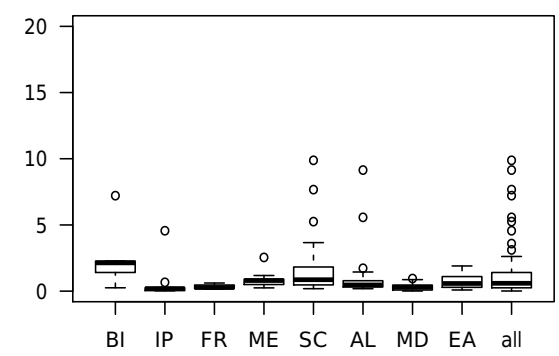

(d) \%sim > perc95 obs JJA (CvM sel)

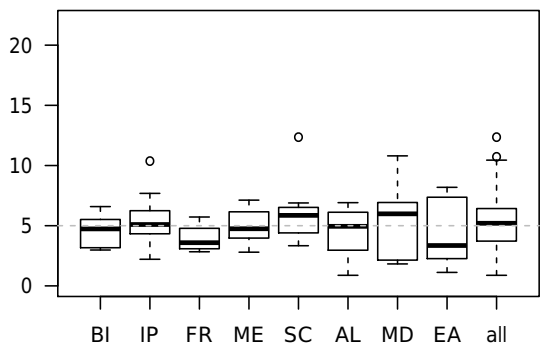

Figure 9. Steps 1 and 2: combined model. Boxplots of (a, b) CvM score and (c, d) percentage of simulated wet days exceeding the observed 95th percentile $\left(\%\right.$ sim $>$ perc95 ${ }_{\text {obs }}$ ) for the cross-validated CvM-selected model. Regions: British Isles (BI), Iberian Peninsula (IP), France (FR), Mid-Europe (ME), Scandinavia (SC), Alps (AL), Mediterranean (MD), and eastern Europe (EA). Note the different scales of the $y$ axes than in Fig. 5. Outliers out of range in (a) ME and all: 22.19; SC and all: 27.12 and 31.35.

the sensitivity of the CvM score is illustrated by Stornoway in JJA (Appendix B3), as this example still yields suitable results despite the relatively high CvM score.

The occurrence of heavy precipitation in the CvM-selected model is slightly overestimated in most subregions in DJF (Figs. 8c and 9c), though quite well represented in IP and FR (Fig. 9c). In JJA heavy precipitation occurrence is quite well estimated (Figs. 8d and 9d). The median of most subregions is very close to $5 \%$ (the "perfect" model would have exactly $5 \%)$. However, some stations, particularly in EA, underestimate the occurrence of heavy precipitation. These are in most cases stations where the VGLM has not been selected, likely indicating problems with the VGLM and the remaining scale gap (see the section before and Sect. 5.2.2).

Ideal performance of our combined model is illustrated in the example QQ plot of Malaga in DJF (Fig. 10); i.e., QM grid corrects the RCM-simulated precipitation on the same scale and the VGLM bridges the remaining scale gap, resulting in a good match of the observations. Sonnblick in DJF (Fig. 10) and JJA (Appendix B3) and Brocken in DJF (Fig. 10) are also well-performing examples. The QQ plot of San Sebastian in DJF (Fig. 10) shows the benefit of selecting the predictor by $\mathrm{CvM}$ as in this case the RCM is used as a predictor for the VGLM. Here using the $\mathrm{QM}_{\text {grid }}$-corrected time series may result in overly high extremes. Sion in JJA (Appendix B3) is another good example of the benefit of model selection where the RCM has been selected as a predictor. Here the high VGLM-simulated quantiles are already overestimated in this setting and would likely be even higher should the $\mathrm{QM}_{\text {grid-corrected predictor be employed. }}$

\subsection{Intercomparison of all models}

In this section an intercomparison of all models (not only the selected best model from Sect. 5.2) for all subregions is presented and compared to the classical application of $\mathrm{QM}_{\text {point }}$. Figure 11 shows boxplots for the CvM score. Generally the cdf bias is lower in JJA than in DJF for all models, already for the uncorrected RCM (apart from BI). In the Mediterranean region (MD and IP) there is a very low cdf bias in all models, indicating general good performance. The QM improves the cdf bias in many regions, with $\mathrm{QM}_{\text {grid }}$ and $\mathrm{QM}_{\text {point }}$ being similar in many cases. The effect of the VGLM depends on region and season. The representation of precipitation is generally improved by the VGLM in BI, IP, AL, and MD in both seasons. However, in FR, ME, SC, and EA in DJF the VGLM introduces biases. The bias increases from west to east ( $\mathrm{FR} \rightarrow \mathrm{ME} \rightarrow \mathrm{EA}$ ) with the largest spatial variability in EA, extending to high CvM values. For continental winter climate the used VGLM gamma model thus appears not to be the ideal model, which suggests that in these regions it may be better to only correct the bias. This raises the question why the results become worse when statistical post-processing is applied. One potential reason for these problems with the VGLM in some regions is that the VGLM gamma is not flexible enough to capture the true predictor-predictand relationship if this relationship is non- 

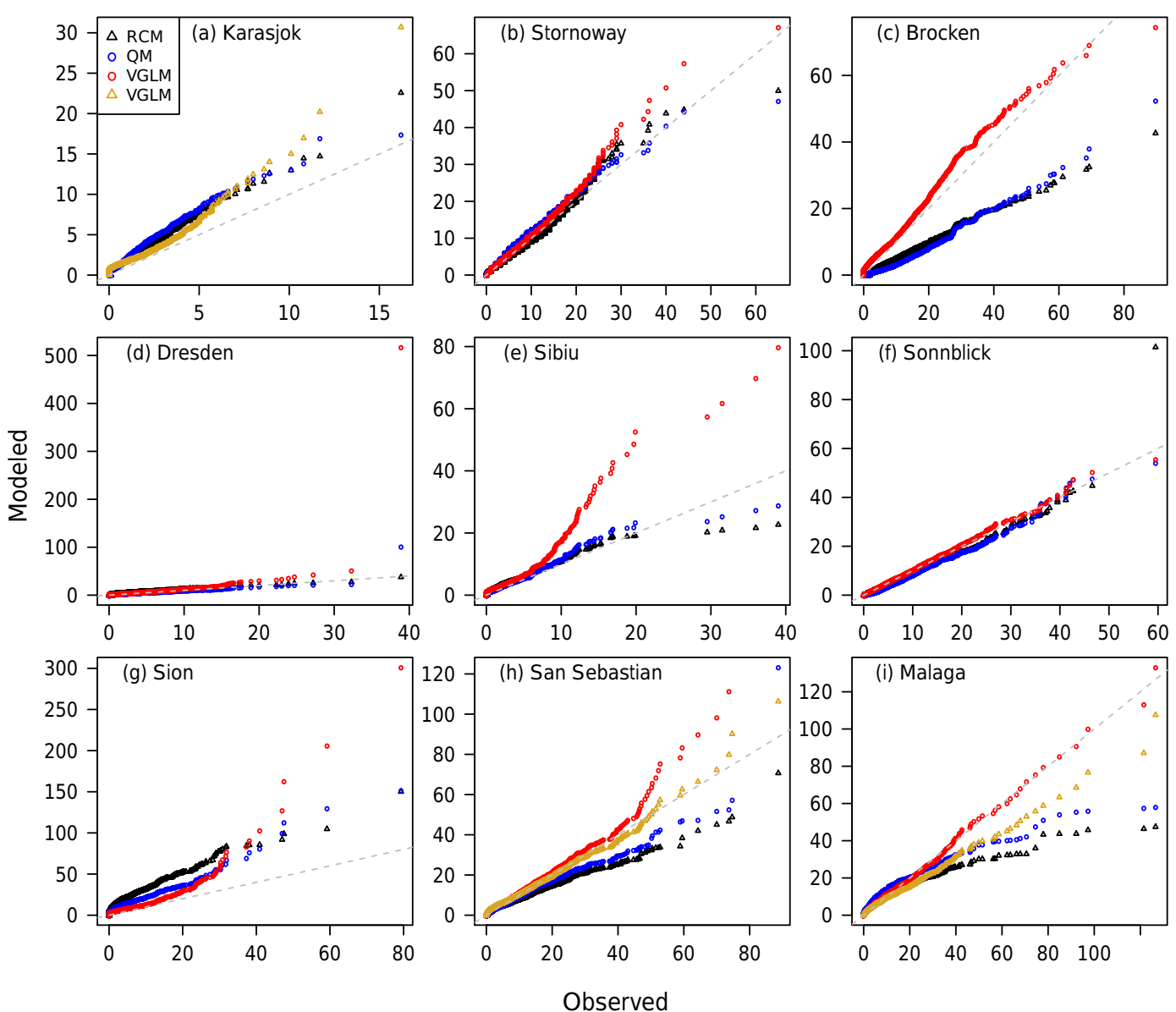

Figure 10. QQ plots for example stations of different models (cross-validated) against station observations for DJF (mm day ${ }^{-1}$ ). (a) Karasjok, (b) Stornoway, (c) Brocken, (d) Dresden, (e) Sibiu, (f) Sonnblick, (g) Sion, (h) San Sebastian, and (i) Malaga. For the VGLM the quantiles (i.e., sorted time series) of 100 realizations are averaged. Predictor for VGLM as selected by the CvM criterion: (red circles) QM $\mathrm{Qmid}_{\text {bias- }}$ corrected RCM; (brown triangles) uncorrected RCM. For examples to illustrate model performance and predictor selection (San Sebastian and Malaga), the VGLM is plotted for both predictors. Selected predictor: San Sebastian: RCM; Malaga: QM grid.

linear as discussed in Sects. 5.2.2 and 5.2.3. The final downscaled marginal distribution may thus be wrong even though it was properly adjusted by the bias-correction step. As the predictor-predictand relationship is always estimated such that it follows well the bulk of the distribution, this problem occurs for predictand values at the very low ends of the VGLM conditional distribution. Furthermore, particularly in EA and FR, E-OBS may be an inappropriate reference for calibration in both QM $_{\text {grid }}$ and VGLM due to low station density. However, in SC stations in E-OBS are relatively dense and, thus, the bias introduced by the VGLM is in that case not attributable to E-OBS quality. In DJF SC has the highest RCM bias among all subregions. This suggests a detailed evaluation of this high bias which is beyond the scope of our study however.

To infer the performance of all studied models in estimating the occurrence of heavy precipitation, boxplots for the percentage of simulated values that are higher than the 95th percentile of the observations on wet days $\left(\%\right.$ sim $>$ perc $\left.95_{\text {obs }}\right)$ for all models are provided in Fig. 12. Particularly in JJA the $\mathrm{QM}_{\text {grid }}$ improves the occurrence of heavy precipitation but remains slightly too dry, which is expected due to the remaining scale gap. The estimated occurrence of heavy precipitation is improved by the VGLM in many cases, although generally slightly overestimated. The results of the VGLM and $\mathrm{QM}_{\text {point }}$ are generally similar, with the $\mathrm{QM}_{\text {point }}$ often being slightly closer to the 5\% line and the VGLM slightly too wet. In AL the VGLM considerably improves the cdf bias (Fig. 11f) and the occurrence of heavy precipitation (Fig. 12f) in both DJF and JJA com-

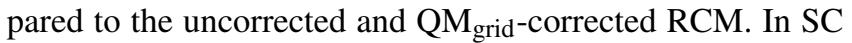
in DJF one should be careful, as although the occurrence of heavy precipitation is considerably improved by the VGLM (Fig. 12e), it introduces biases when the whole cdf is evaluated (Fig. 11e), and is thus not recommended. Concerning heavy precipitation occurrence our model shows a similar 

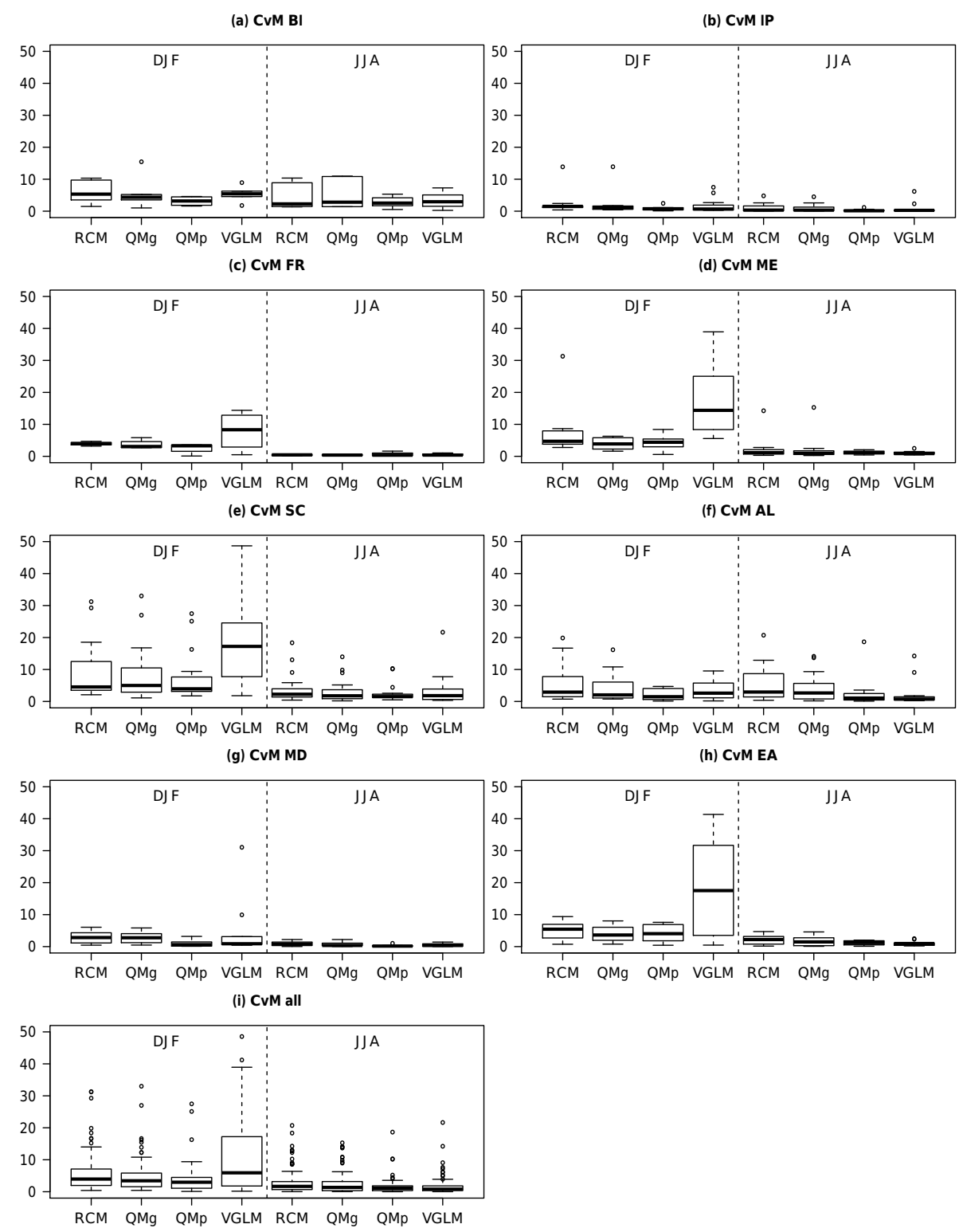

Figure 11. Intercomparison of all cross-validated models (not only the selected best model). Models: uncorrected $\mathrm{RCM}^{\mathrm{Q}} \mathrm{QM}$ grid-corrected RCM to grid scale, $\mathrm{QM}_{\text {point }}$-corrected RCM to point scale, and predictor and VGLM-downscaled RCM. Boxplots of the CvM score for all models in different subregions: (a) British Isles (BI), (b) Iberian Peninsula (IP), (c) France (FR), (d) Mid-Europe (ME), (e) Scandinavia (SC), (f) Alps (AL), (g) Mediterranean (MD), (h) eastern Europe (EA), and (i) all locations. For the VGLM the CvM score was computed for 100 realizations and then averaged. Outlier out of range in (d, i) RCM DJF: 54.19.

behavior for all subregions in JJA and for IP also in DJF (Fig. 12). The $\mathrm{QM}_{\text {grid }}$ bias correction improves the representation but remains too dry. The dry bias is then eliminated by the VGLM though to slightly too many "extremes". This model behavior as exhibited in JJA is exactly what would be expected due to the scale gap between gridded and point scales. Due to more small-scale convective extremes this scale gap has a larger impact in summer, whereas in winter most extremes are caused by large-scale weather systems that are generally better represented by the gridbox scale, also at coarser resolutions. While the cdf bias and the occurrence of heavy precipitation reveal how well properties of the precipitation distribution are represented, they do not allow us to draw conclusions about the predictive power of the model.

To infer whether our model has predictive power, we cannot assess temporal correspondence compared to observations as in Wong et al. (2014) and Eden et al. (2014) because we use an RCM that is not nudged, and even though driven with perfect boundary conditions (reanalysis) this is not a clean pairwise setup. Instead, we evaluate spatial au- 
$\begin{array}{ll}\text { (a) \%sim > perc95 obs BI } & \text { (b) \%sim > perc95 obs IP }\end{array}$
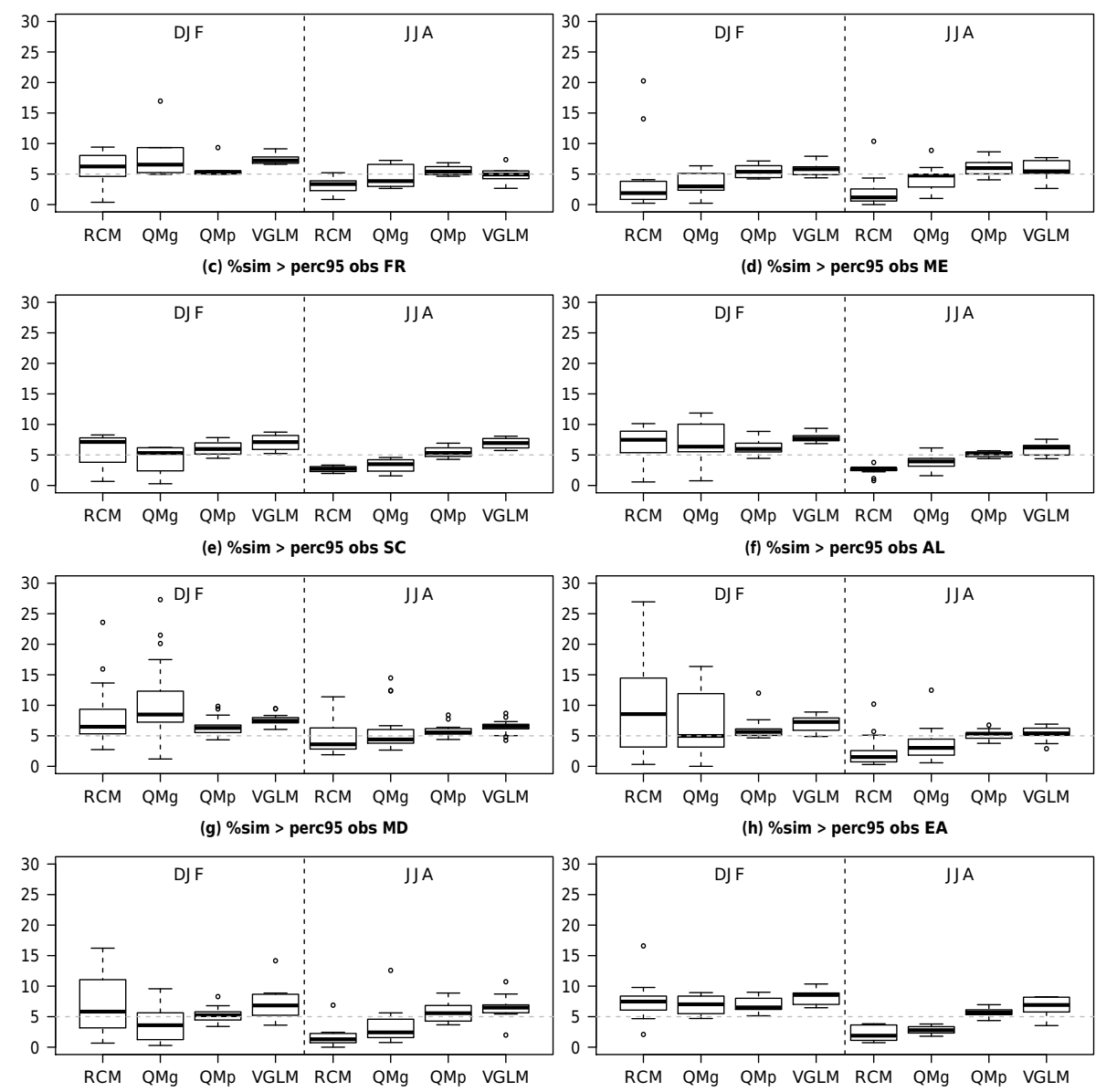

(h) \%sim > perc95 obs EA
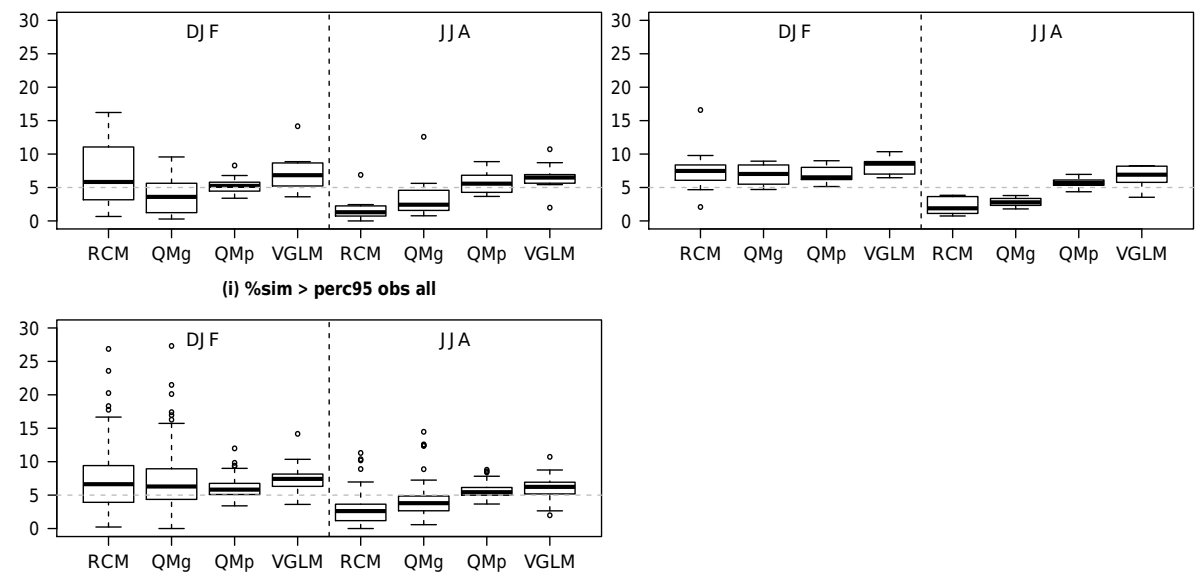

Figure 12. As in Fig. 11, but for percentage of simulated wet days exceeding the observed 95 th percentile $\left(\%\right.$ sim $>$ perc $\left.95_{\text {obs }}\right)$. Outlier out of range in $\left(\mathbf{g}\right.$, i) $\mathrm{QM}_{\text {grid }}$ DJF: $41.07 \%$.

tocorrelation, which is the correlation of a variable with itself in geographical space. This allows us to evaluate whether the model correctly reproduces daily spatial autocorrelations and, thus, the spatial extent of precipitation patterns, including its variability in time compared to observed precipitation. In Fig. 13 correlograms of the cross-validated time series of all models (RCM, $\mathrm{QM}_{\text {grid }}, \mathrm{QM}_{\text {point }}, 100$ VGLM realizations) and station observations as a reference are provided. The spatial autocorrelation of QM-bias-corrected precipitation decays very similarly to uncorrected RCM precipitation and thus shows only little improvement of spatial autocorrelation compared to point-scale observations. Differences between $\mathrm{QM}_{\text {grid }}$ and $\mathrm{QM}_{\text {point }}$ are negligible. This confirms that the QM approach is not capable of modeling small-scale variability, and a stochastic model is thus needed to bridge the scale gap. The spatial autocorrelation of VGLM-downscaled precipitation decays more similarly to the station observations than the QM corrected or uncorrected RCM, particularly in JJA. The spatial dependence is thus improved by the stochastic downscaling step. The long decorrelation length in DJF is underestimated by our stochastic, single-site model, which indicates a slightly too strong noise component. A spatial model considering more than one station or including more physically based predictors (i.e., sea level pressure) might improve the predictive power of our model in DJF. 
(a) DJF

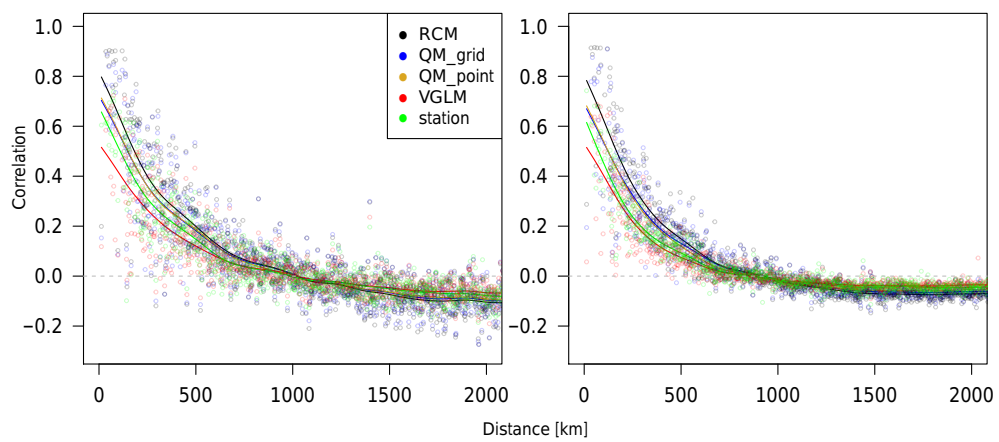

Figure 13. Spatial autocorrelation (cross-validated). Correlogram (circles) and smoothed spline fitted to the correlogram (lines) for (a) DJF and (b) JJA. The correlogram is estimated by the centered Mantel statistic using R package ncf (Bjornstad, 2015). For the VGLM 100 realizations of the stochastic model for each station were used to estimate the correlogram.

\section{Conclusions}

We introduced the concept of a combined statistical bias correction and stochastic downscaling method for precipitation. We thereby extend the stochastic model output statistics (MOS) approach developed by Wong et al. (2014) beyond nudged simulations to free-running GCM/RCM simulations. We applied our method to precipitation simulated by the RCM KNMI-RACMO2 driven with ERA-Interim boundary conditions within the EURO-CORDEX framework. As the RCM is driven with reanalysis we only correct RCM biases. Our method corrects the "drizzle effect" (i.e., too many wet days), overly low precipitation values in the rain shadows caused by not enough windward air masses crossing the mountain range ("location bias", Maraun and Widmann, 2015), and precipitation intensity. To correct the "drizzle effect" we increased the wet day threshold such that the number of wet days (closely) matches the gridded observations with a threshold of $0.1 \mathrm{~mm} \mathrm{day}^{-1}$ (Maraun, 2016). To overcome the "location bias" we selected the RCM gridbox that best represents the climate in the respective gridbox of the gridded observations (Maraun and Widmann, 2015). Note that when transferring the approach to free-running simulations this gridbox selection step has to be calibrated with a reanalysis-driven simulation of the RCM to ensure temporal correspondence. Consequently, only the location bias caused by the RCM is corrected. How a potential location bias of the driving GCM may affect the results should be analyzed in future work. Precipitation intensities were corrected by a parametric quantile mapping (QM) approach between RCM and gridded observations on the same spatial scale. As precipitation is highly variable in space and time, not all variability can be explained by the gridbox scale (Maraun, 2013a). To bridge the gap between gridbox and point scale we applied a stochastic regression-based model. For evaluation we adopted the experimental framework of VALUE (Maraun et al., 2015). In this context, we applied our method to 86 example rain gauges across Europe representing differ- ent climates, and carried out a 5-fold cross-validation for the time period 1979-2008. Both steps of the combined method were evaluated individually and combined. A comparison to classical QM between RCM and point scale is also provided.

The proposed parametric model structure appears not to be the optimal choice for all considered stations. Yet given that the aim of our study is a proof of concept, the identification of an optimal model for all individual cases would be beyond the scope of this work. Nevertheless, where our implementation is not adequate we provide suggestions for improvements within the presented framework. Our specific implementation for the QM bias correction (first step) of wet day intensities employs the mixture distribution of a gamma distribution for the precipitation mass and a generalized Pareto (GP) distribution for the extreme tail (Frigessi et al., 2002; Vrac and Naveau, 2007). The stochastic regressionbased model for downscaling (second step) was calibrated between observations on gridded and point scales, and then transferred to bias-corrected RCM-simulated precipitation. This corresponds to a perfect prog (PP) approach. The regression model consists of a logistic regression to model wet day probabilities and a vector generalized linear model (VGLM) predicting the parameters of a gamma probability distribution for precipitation intensities. The QM-corrected time series (first step) was used as a predictor for downscaling (second step) if it improves the representation of precipitation compared to the uncorrected RCM. Thus, we selected the predictor based on the lower cdf bias by applying the CvM criterion with the gridded E-OBS dataset as a reference.

Precipitation was in most cases improved by (parts of) our combined method across different European climates; to what extent depends on region and season though. The method generally performs better in JJA than in DJF and in DJF best in the Mediterranean region, with a mild winter climate, and worst for the continental winter climate in Midand eastern Europe or Scandinavia. Seasonal and regional differences depending on the underlying mechanism have al- 
ready been reported for resolution dependence of extreme precipitation in GCMs (Volosciuk et al., 2015) and RCMs (Prein et al., 2013; Meredith et al., 2015). Hence, for a good representation of precipitation extremes, the complexity of the model can be chosen at each step of the modeling cascade based on the underlying mechanism in order to use computational resources efficiently.

Although our bias correction (first step) improved simulated precipitation for many locations in both seasons, wet biases may remain even after bias correction, particularly for continental winter. In agreement with our results, large improvements by bias correction over the Alps, Spain, and France have been reported by Dosio and Paruolo (2011). However, in contrast to our results these authors also obtain good results for Mid- and eastern Europe, where we find persisting biases even after bias correction. In the cases where the quantile mapping approach does not improve RCMsimulated precipitation, another transfer function might be more suitable. Choosing between different parametric transfer functions as proposed by Piani et al. (2010) could improve the results. By employing a quantile mapping approach we presumed both a stationary statistical relationship and stationary cdfs that also apply in a changed future climate. However, in a climate change context RCM-simulated trends in the cdf are modified by applying such statistical postprocessing. For cases where the GCM/RCM simulates plausible climate change trends the CDF-t concept suggested by Michelangeli et al. (2009) and Vrac et al. (2012) might be an appropriate framework. In their concept the correction function explicitly accounts for future trends in the RCMsimulated distribution. Thereby simulated trends in all moments are approximately preserved after bias correction. For instance, regions where an increase in extreme precipitation accompanied by a decrease in mean precipitation is projected (e.g., in central European summer, Christensen and Christensen, 2003; Maraun, 2013b), these trends might be better represented by employing a CDF-t method. However, in this study we have not employed this variant as in our setting the validation period is too short to achieve an appropriate fit of the future mixture distribution. Quantifying the differences between the quantile mapping approach we employed here and a CDF-t approach is left for future work when our combined method will be applied to climate change scenarios.

The stochastic downscaling (second step) improves the estimated occurrence of heavy precipitation in many regions, but introduces biases in continental winter climate. Furthermore, spatial autocorrelation in JJA is improved by the VGLM, showing the importance of randomization in the framework of downscaling as already pointed out by, e.g., von Storch (1999) and Maraun (2013a). Moreover, when downscaling climate change scenarios the randomization component of the VGLM that adds small-scale unexplained variability does not modify trends, in contrast to purely deterministic methods, e.g., QM (Maraun, 2013a). However, the deterministic part of the VGLM that corrects system- atic local effects (e.g., lee/windward side of a mountain) alters the pdf, and may thus also change trends. The stochastic downscaling step is more important in JJA than in DJF for both estimation of heavy precipitation occurrence and spatial autocorrelation. This can be attributed to the different underlying main mechanism for heavy precipitation. In summer heavy precipitation is often caused by small-scale convective events, whereas in winter large-scale weather systems dominate. Hence, there is less small-scale variability unexplained by the gridbox in DJF. In DJF spatial autocorrelation is slightly underestimated by the VGLM, which is likely related to the long decorrelation length of precipitation in winter that is not correctly represented in our singlesite model, indicating a slightly too strong noise component. An extension of our method to a multi-site model and/or including more physically based predictors (i.e., sea level pressure) would likely improve this feature and can be the subject of future work. A possible extension to multi-variate or full fields might be based on copulas (e.g., Ferraris et al., 2003; Schölzel and Friederichs, 2008; Bárdossy and Pegram, 2009) or random cascade models (Thober et al., 2014). A good representation of the mild climate in the British Isles is consistent with Wong et al. (2014) and Eden et al. (2014). In France, Mid-Europe, eastern Europe, and Scandinavia in DJF the VGLM introduces biases, raising the question why the results become worse when statistical post-processing is applied. Particularly in France and eastern Europe the E-OBS gridded observational dataset may be an unreliable reference for model calibration for both the QM and the VGLM due to low station density. The "true" resolution of E-OBS in these regions might be coarser than the resolution it is gridded to. This highlights the fact that the applicability of our method is limited to regions where high-quality gridded datasets are available. However, a detailed evaluation of the sensitivity of our method to station density in the gridded dataset is beyond the scope of this study. The bias introduced by the VGLM generally increases from west to east, and, thus, from maritime to continental winter climate. However, in Scandinavia the VGLM also introduces biases even though station density is high. This indicates that although the quality of the E-OBS data may contribute to these problems, it can not be identified as the main source of error. It is rather one potential reason among others. For instance, in some cases the generalized linear relationship between the predictor and the parameters of the gamma distribution appears to be not flexible enough to capture the true predictor-predictand relationship, which can be nonlinear, particularly in but not restricted to continental winter climate. In these regions there may be a more adequate parametric relationship than our specific implementation. Problems with the current implementation may be related to, e.g., the linear structure of the model or the choice of the link function. For instance, another distribution in the VGLM (e.g., mixture model), splines as applied in Maraun et al. (2011b) or a vector generalized additive model (VGAM, Yee and Wild, 1996), are potential ap- 
proaches. However, employing a more complex model also comes with the risk of overfitting. Finding the optimal model for each of the analyzed stations is beyond the scope of this study however.

The varying performance of our specific implementation clearly shows that bias correction and downscaling methods should be reevaluated when transferring them to locations with different climatic conditions. In some regions a specific implementation different from the one we used is required. We recommend our model in summer for all studied regions. However, in winter it should only be used for the British Isles, the Alps, the Mediterranean region, and the Iberian Peninsula, but not for continental winter climates (Scandinavia, Mid-Europe, and eastern Europe) and France. While the stochastic downscaling step (VGLM) is very important for representing spatial autocorrelation in summer, it is less important in winter, where the application of solely the biascorrection step might be sufficient. The concept can generally be extended to a wide range of method combinations. Transferring this concept to other climate variables should in principle be possible. Our specific implementation should be applicable to any gamma-distributed variable. However, our approach has so far only been evaluated for precipitation. Thus, users need to evaluate the model for the particular variable at the chosen location when transferring it.

We developed our model in the present-day climate. In a climate change context the model does not explicitly modify climate trends on a physical basis. Our model is thus only applicable where changes are correctly simulated by the GCM/RCM. For instance, changes in the dynamics of local extreme convective events in summer that need even higher resolution up to convection-permitting simulations (e.g., Kendon et al., 2014; Chan et al., 2014; Meredith et al., 2015) will also not be represented after statistical postprocessing is applied. Bias correction and (dynamical and statistical) downscaling of precipitation is only applicable if the large-scale patterns and changes therein are simulated reasonably by the driving GCM (Eden et al., 2012; Hall, 2014). Therefore, when transferring our method to a GCM or GCM-driven RCM the relevant processes for precipitation in the studied region need to be correctly simulated. For instance, biases in simulated precipitation related to biases in the storm track (Chang et al., 2012), El Niño-Southern Oscillation (ENSO; Zhang and Sun, 2014), the monsoon (Hasson et al., 2013), or persistent weather regimes (Petoukhov et al., 2013; Palmer, 2013) cannot be statistically corrected in a physically sensible way.
The general concept of combining two methods and thereby separating bias correction (MOS) and downscaling (PP) into two steps is a powerful approach as it benefits from the respective methodological advantages. Additionally, the strength of this two-step method is that the best combination of methods can be selected. This implies that the concept can be extended to a wide range of method combinations.

Data availability. The RCM output from the KNMI that was used in this study is available within the CORDEX framework from the Earth system grid federation (e.g., https://esgf-data.dkrz.de/ projects/esgf-dkrz/) upon registration. The E-OBS gridded dataset is available at the ECA\&D website (http://www.ecad.eu/download/ ensembles/download.php). The ECA\&D station data are provided by the KNMI on the ECA\&D website (http://www.ecad.eu/ dailydata/customquery.php). The specific selection for the VALUE experiment can be downloaded from the VALUE website (http: //www.value-cost.eu/data). Bias-corrected and downscaled data as well as the source code from this study are available from the authors upon request. 
Appendix A: Technical details for bias-correction implementation and model selection

\section{A1 Technical details for model implementation}

A non-zero wet day threshold assigns zero probability density to all intensities between zero and the threshold, resulting in a misfit of the gamma distribution (Wong et al., 2014). To avoid this we shift precipitation on all wet days by subtracting the threshold for calibration. The estimated distribution is subsequently shifted back by the threshold.

Numerical instabilities in the estimation of the mixture cdf may in rare cases result in a discontinuous cdf (Fig. A1a). In these cases we interpolate linearly between the continuous probabilities surrounding the discontinuity. The example cdf in Fig. A1a illustrates that this procedure is a reasonable estimation for these quantiles. If the cdf does not "jump back" as in Fig. A1 a but continues as illustrated in Fig. A1b, the model has to be sorted out as there is no straightforward possibility of handling this artifact caused by numerical instability. However, the latter case only occurs extremely rarely.

\section{A2 Technical details for model selection}

The AIC performs best for the part of the distribution where most of the values are. Hence, a good fit for the bulk of the distribution might include large biases in the extremes and still have the lowest AIC (example: Fig. A1c). To avoid such a model choice with unreasonable high extremes, we introduce a criterion based on the extremes to sort out mixture model fits yielding overly high extremes before AIC-model selection is applied. This criterion is based on a comparison between the 100 season return level estimated by the mixture model (RL100S mixture $_{\text {) }}$ and the $95 \%$ confidence interval of the RL100S ${ }_{\mathrm{GP}}$ estimated by the GP distribution only. The RL100S $\mathrm{SP}_{\mathrm{GP}}$ and the corresponding $95 \%$ confidence interval are estimated according to Coles (2001). This criterion is applied differently for $F_{\mathrm{obs}}$ and $F_{\mathrm{RCM}}$ considering the respective relevant quantity for the correction function. For $F_{\text {obs }}$ this criterion is based on the return level itself, whereas for $F_{\mathrm{RCM}}$ the probability for the return level is considered. In particular, for $F_{\text {obs }}$ the RL100S mixture must not exceed the $95 \%$ confidence interval of the RL100S ${ }_{\mathrm{GP}}$. For $F_{\mathrm{RCM}}$ the mixture model probability ( $p_{\text {mixture }}$ ) for the RL100S ${ }_{\mathrm{GP}}$ must not exceed $p_{\mathrm{GP}}$ for the $95 \%$ confidence interval of RL100S $_{\mathrm{GP}}$. Furthermore, $p_{\text {mixture }}$ for the $95 \%$ confidence interval of the RL100S $\mathrm{GP}$ must not be very close to 1 (i.e., $>1-1 \times 10^{-15}$ ) as a reasonable extrapolation to potentially higher values under climate change would not be possible in that case. (a) Numerical instability
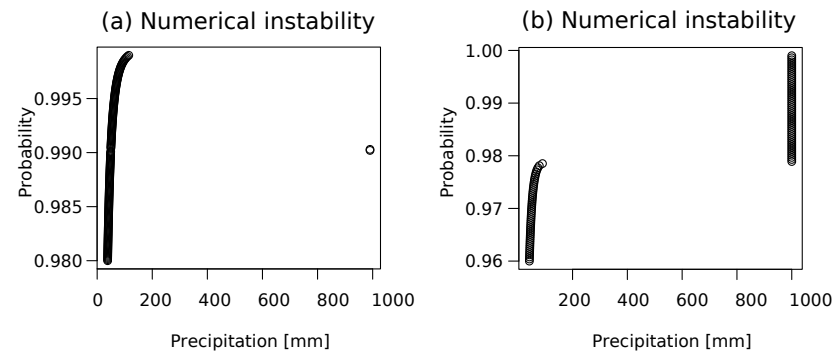

(c) Model selection problem

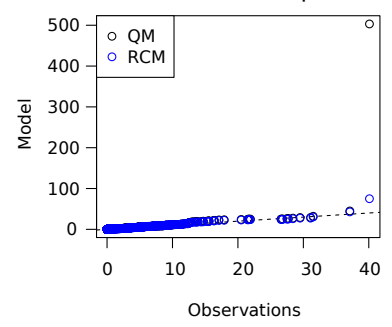

Figure A1. Examples of problems with the mixture model. (a) $\mathrm{Nu}-$ merical instability: discontinuous cdf; (b) numerical instability: cdf that jumps to the upper bound of $1000 \mathrm{~mm} \mathrm{day}^{-1}$ and does not jump back as in (a), and (c) problematic model selection: QQ plot of a selected mixture model that fits well for most quantiles but corrects the extremes to too wet. 
Appendix B: Additional results

B1 Step 1: bias correction

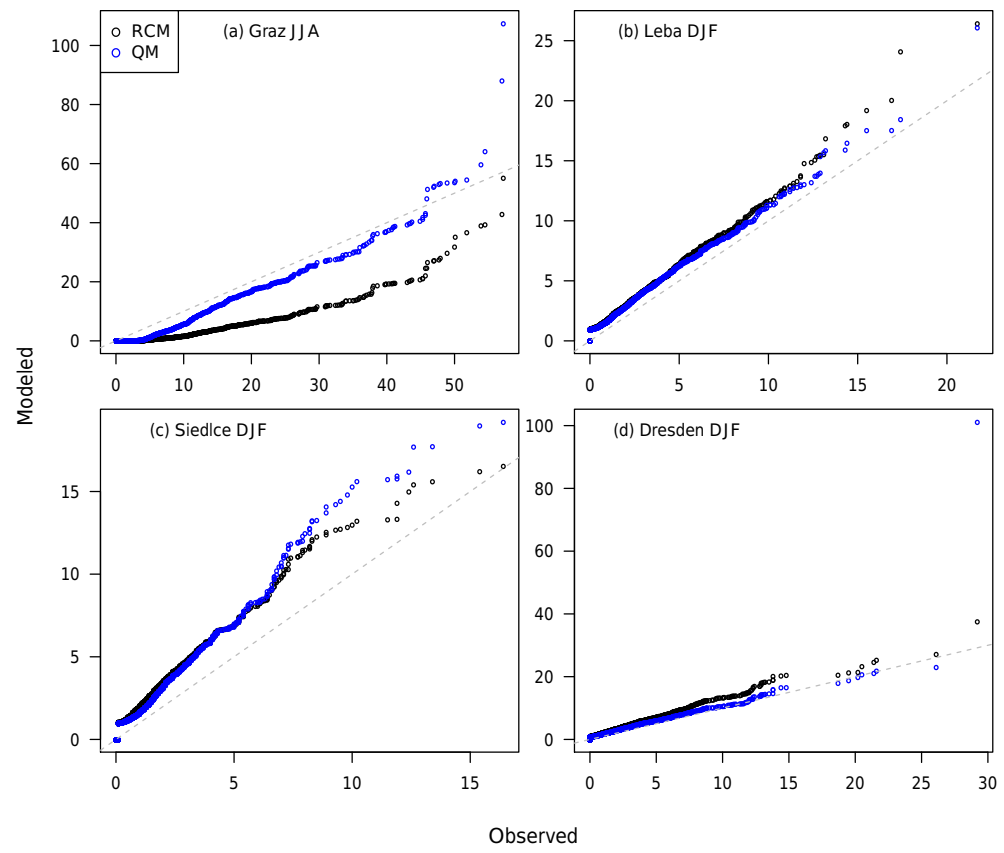

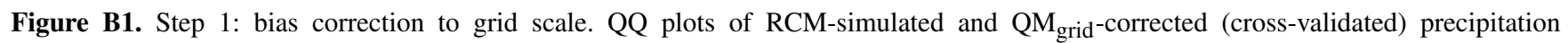
$\left(\mathrm{mm} \mathrm{day}^{-1}\right.$ ) against E-OBS for stations with a high CvM score. (a) Graz JJA, (b) Leba DJF, (c) Siedlce DJF, and (d) Dresden DJF. 
B2 Step 2: downscaling
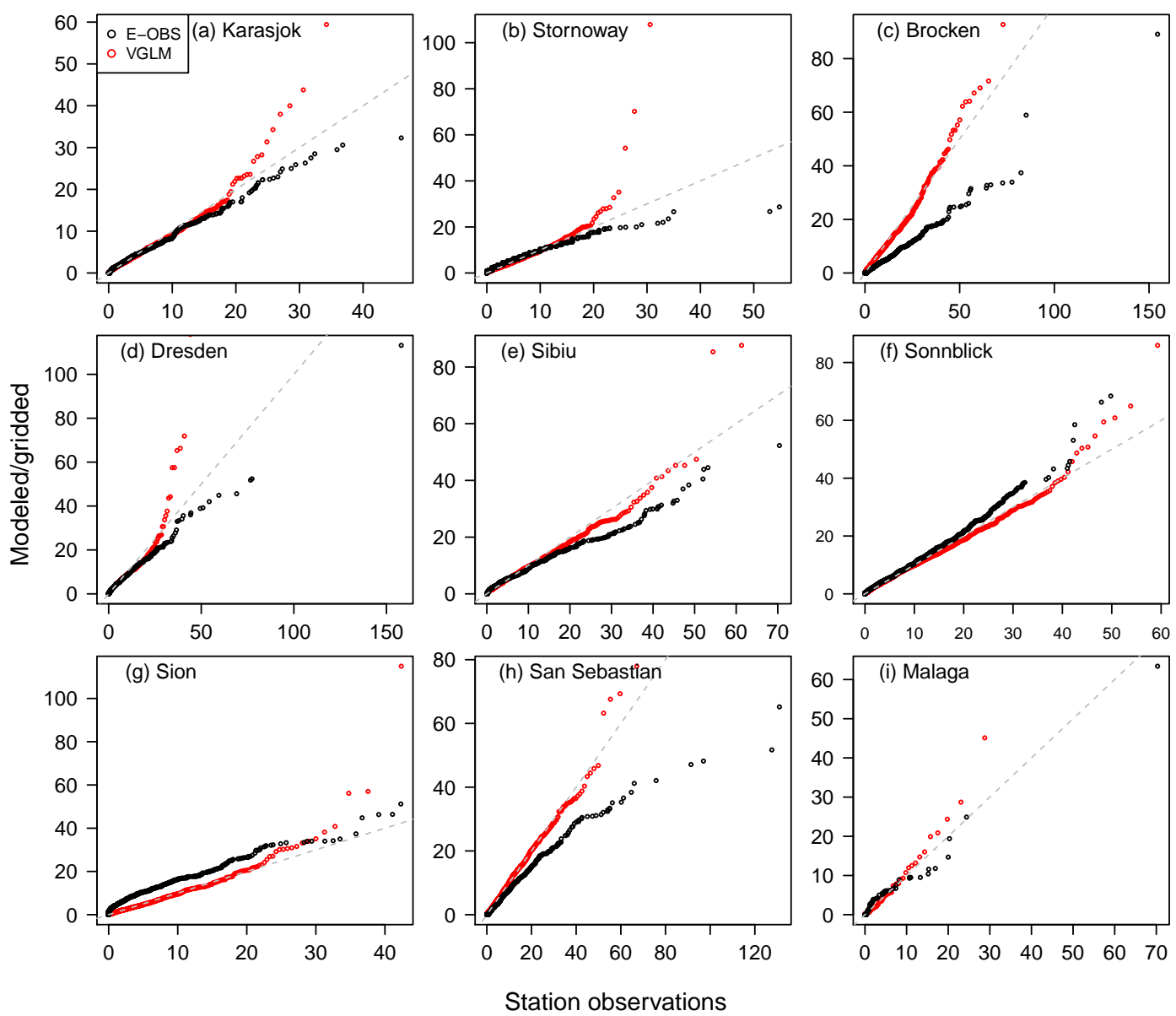

Figure B2. Step 2: downscaling. QQ plots for example stations in JJA. VGLM gamma standardized to the stationary gamma distribution fitted to observed wet day intensities between gridded and point-scale precipitation observations (mm day ${ }^{-1}$ ). (a) Karasjok, (b) Stornoway, (c) Brocken, (d) Dresden, (e) Sibiu, (f) Sonnblick, (g) Sion, (h) San Sebastian, and (i) Malaga. 

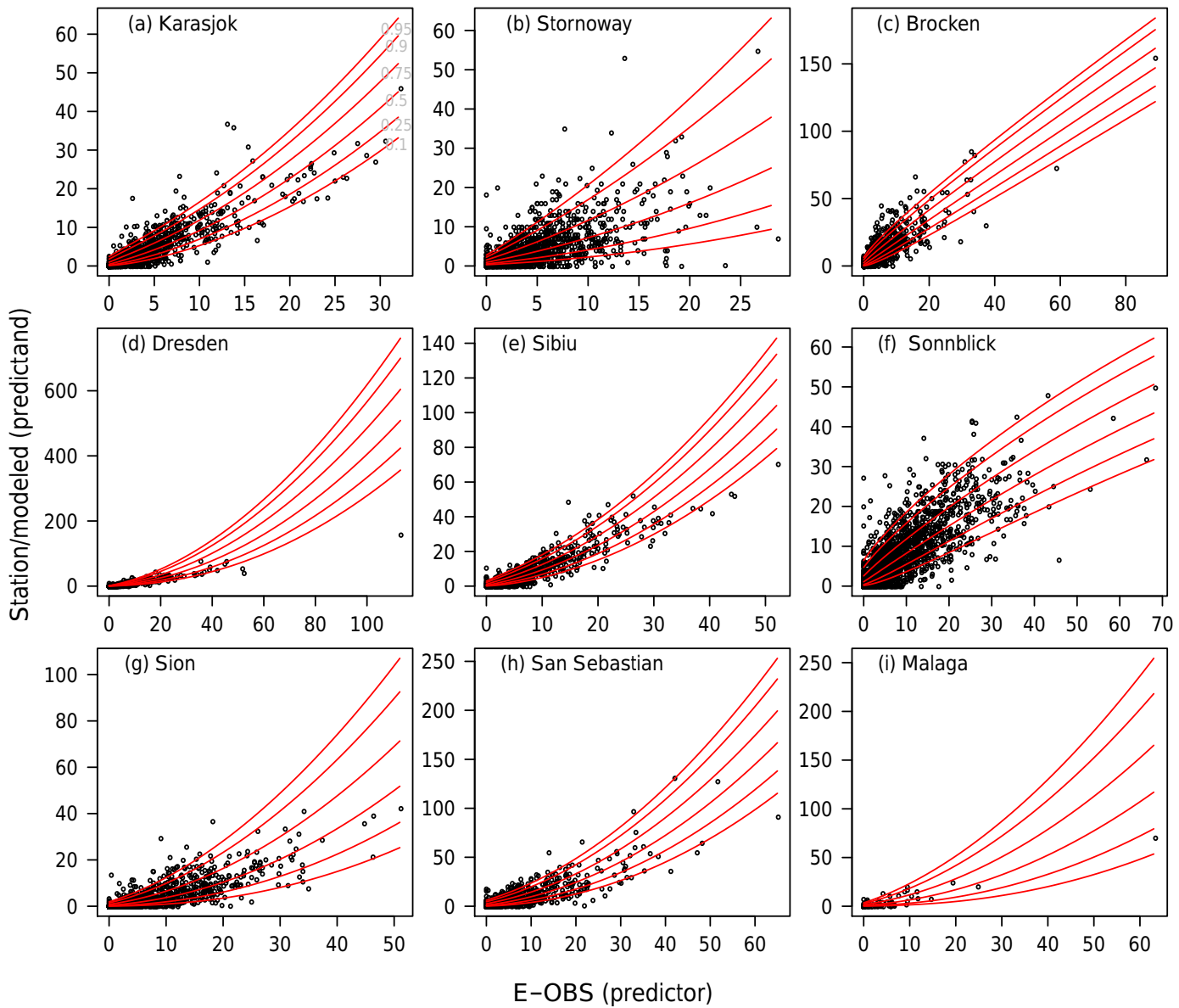

Figure B3. Step 2: downscaling. Estimated relation between gridded and point-scale precipitation observations for example stations in JJA. VGLM gamma where both parameters depend on the predictor fitted to observed wet day intensities. The predictor is E-OBS. Circles: observed precipitation intensities $\left(\mathrm{mm} \mathrm{day}^{-1}\right)$; lines: $0.1,0.25,0.5,0.75,0.9$, and 0.95 modeled quantiles $\left(\mathrm{mm} \mathrm{day}^{-1}\right)$. (a) Karasjok, (b) Stornoway, (c) Brocken, (d) Dresden, (e) Sibiu, (f) Sonnblick, (g) Sion, (h) San Sebastian, and (i) Malaga. 
B3 Combination of steps 1 and 2: bias correction and downscaling
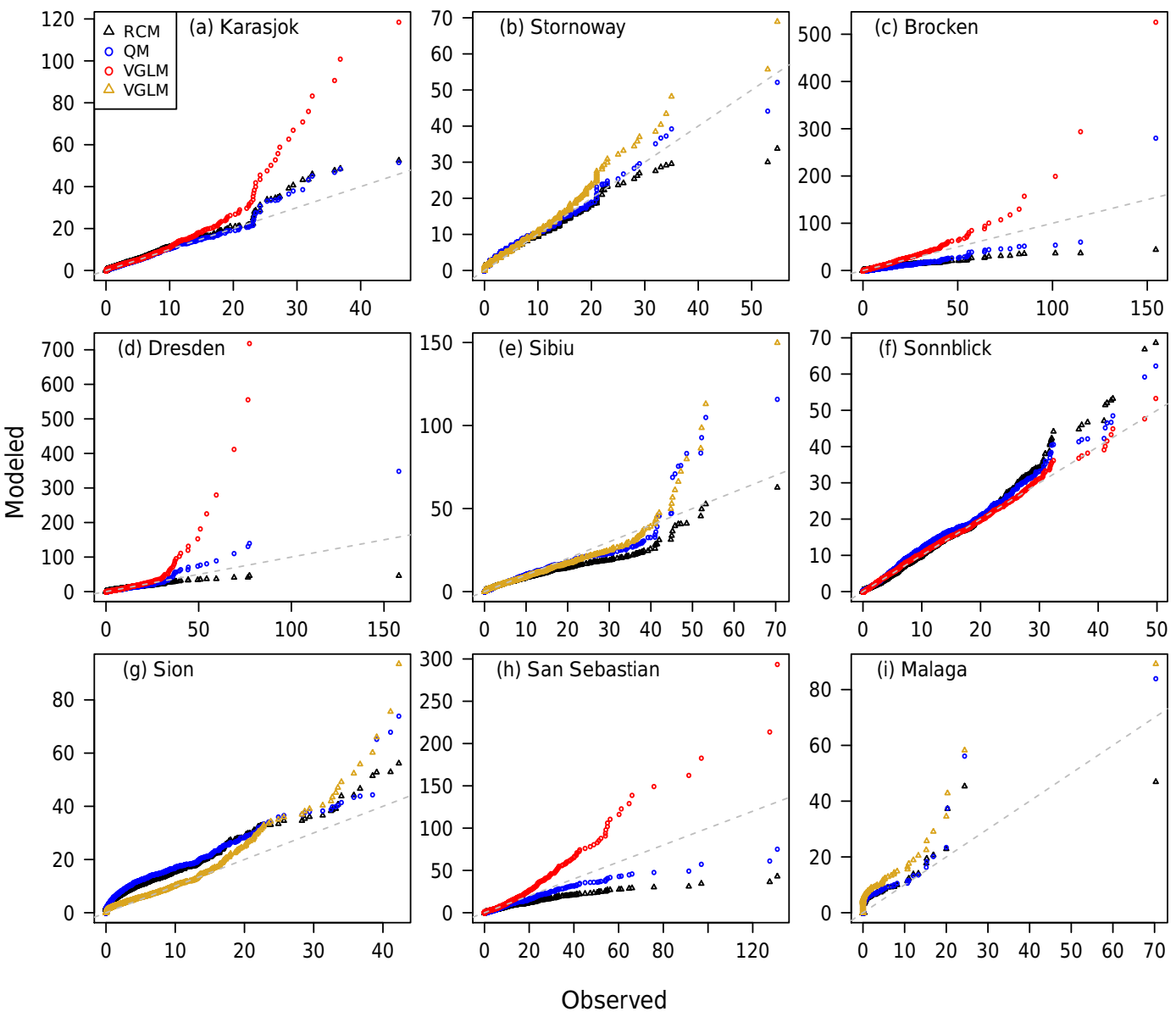

Figure B4. QQ plots for example stations of different models (cross-validated) against station observations for JJA (mm day ${ }^{-1}$ ). (a) Karasjok, (b) Stornoway, (c) Brocken, (d) Dresden, (e) Sibiu, (f) Sonnblick, (g) Sion, (h) San Sebastian, and (i) Malaga. For the VGLM the quantiles (i.e., sorted time series) of 100 realizations are averaged. Predictor for VGLM as selected by the CvM criterion: (red circles) $\mathrm{QM}_{\text {grid }}$ biascorrected RCM, (brown triangles) uncorrected RCM. Highest VGLM-modeled quantile in Dresden out of range: $3609 \mathrm{~mm} \mathrm{day}^{-1}$. 
Author contributions. Douglas Maraun had the initial idea for this combined method. Claudia Volosciuk implemented the method and performed the evaluation with help from Mathieu Vrac and Douglas Maraun. All authors discussed details of the implementation and the results. Claudia Volosciuk prepared the manuscript with contributions from all co-authors.

Competing interests. The authors declare that they have no conflict of interest.

Acknowledgements. We thank the KNMI for producing and making available their model output. We acknowledge the E-OBS dataset from EU-FP6 project ENSEMBLES (http://ensembles-eu.metoffice.com) and the data providers in the ECA\&D project (http://eca.knmi.nl). We thank S. Kotlarksi, S. Hagemann, and one anonymous reviewer for comments on the manuscript. The analysis was carried out with $\mathrm{R}$, using the packages evir, ncdf, MASS, stats, stats4, fields, aspace, ncf, and rworldmap. This study was funded by the EUREX project of the Helmholtz Association (HRJRG-308) and the PLEIADES project of the Volkswagen Foundation (grants 85423 and 85425). Claudia Volosciuk has received a Short-Term Scientific Mission Grant from EU COST Action ES1102 VALUE.

The article processing charges for this open-access publication were covered by a Research

Centre of the Helmholtz Association.

Edited by: L. Samaniego

Reviewed by: S. Kotlarski, S. Hagemann, and one anonymous referee

\section{References}

Ahmed, K. F., Wang, G., Silander, J., Wilson, A. M., Allen, J. M., Horton, R., and Anyah, R.: Statistical downscaling and bias correction of climate model outputs for climate change impact assessment in the U.S. northeast, Global Planet. Change, 100, 320332, doi:10.1016/j.gloplacha.2012.11.003, 2013.

Akaike, H.: Information theory and an extension of the maximum likelihood principle, in: Proc. Second Int. Symp. on Information Theory, Institute of Electrical and Electronics Engineers, Budapest, Hungary, 267-281, 1973.

Bárdossy, A. and Pegram, G. G. S.: Copula based multisite model for daily precipitation simulation, Hydrol. Earth Syst. Sci., 13, 2299-2314, doi:10.5194/hess-13-2299-2009, 2009.

Bjornstad, O. N.: ncf: Spatial nonparametric covariance functions, r package version 1.1-6, http://CRAN.R-project.org/package= ncf (last access: 17 March 2017), 2015.

Chan, S. C., Kendon, E. J., Fowler, H. J., Blenkinsop, S., and Roberts, N. M.: Projected increases in summer and winter UK sub-daily precipitation extremes from high-resolution regional climate models, Environ. Res. Let., 9, 084019, doi:10.1088/1748-9326/9/8/084019, 2014.

Chandler, R. E. and Wheater, H. S.: Analysis of rainfall variability using generalized linear models: A case study from the west of Ireland, Water Resour. Res., 38, 1192, doi:10.1029/2001WR000906, 2002.

Chang, E. K. M., Guo, Y., and Xia, X.: CMIP5 multimodel ensemble projection of storm track change under global warming, J. Geophys. Res.-Atmos., 117, D23118, doi:10.1029/2012JD018578, 2012.

Christensen, J. H. and Christensen, O. B.: Severe summertime flooding in Europe, Nature, 421, 805-806, doi:10.1038/421805a, 2003.

Christensen, J. H. and Christensen, O. B.: A summary of the PRUDENCE model projections of changes in European climate by the end of this century, Climatic Change, 81, 7-30, doi:10.1007/s10584-006-9210-7, 2007.

Coles, S.: An introduction to statistical modeling of extreme values, Springer-Verlag, London, 2001.

Darling, D.: The Kolmogorov-Smirnov, Cramér-von Mises tests, Ann. Math. Stat., 28, 823-838, 1957.

Dee, D. P., Uppala, S. M., Simmons, A. J., Berrisford, P., Poli, P., Kobayashi, S., Andrae, U., Balmaseda, M. A., Balsamo, G., Bauer, P., Bechtold, P., Beljaars, A. C. M., van de Berg, L., Bidlot, J., Bormann, N., Delsol, C., Dragani, R., Fuentes, M., Geer, A. J., Haimberger, L., Healy, S. B., Hersbach, H., Hólm, E. V., Isaksen, L., Kållberg, P., Köhler, M., Matricardi, M., McNally, A. P., Monge-Sanz, B. M., Morcrette, J.-J., Park, B.-K., Peubey, C., de Rosnay, P., Tavolato, C., Thépaut, J.-N., and Vitart, F.: The ERA-Interim reanalysis: configuration and performance of the data assimilation system, Q. J. Roy. Meteorol. Soc., 137, 553597, doi:10.1002/qj.828, 2011.

Dobson, A. J.: An introduction to generalized linear models, 2nd Edn., Chapman and Hall, Boca Raton, Florida, 2001.

Dosio, A. and Paruolo, P.: Bias correction of the ENSEMBLES high-resolution climate change projections for use by impact models: Evaluation on the present climate, J. Geophys. Res.Atmos., 116, d16106, doi:10.1029/2011JD015934, 2011.

Eden, J. M., Widmann, M., Grawe, D., and Rast, S.: Skill, correction, and downscaling of GCM-simulated precipitation, J. Climate, 25, 3970-3984, doi:10.1175/JCLI-D-11-00254.1, 2012.

Eden, J. M., Widmann, M., Maraun, D., and Vrac, M.: Comparison of GCM and RCM simulated precipitation following stochastic postprocessing, J. Geophys. Res.-Atmos., 119, 11040-11053, doi:10.1002/2014JD021732, 2014.

Ferraris, L., Gabellani, S., Rebora, N., and Provenzale, A.: A comparison of stochastic models for spatial rainfall downscaling, Water Resour. Res., 39, 1368, doi:10.1029/2003WR002504, 2003.

Flato, G., Marotzke, J., Abiodun, B., Braconnot, P., Chou, S., Collins, W., Cox, P., Driouech, F., Emori, S., Eyring, V., Forest, C., Gleckler, P., Guilyardi, E., Jakob, C., Kattsov, V., Reason, C., and Rummukainen, M.: Evaluation of Climate Models, in: Climate Change 2013: The Physical Science Basis, Contribution of Working Group I to the Fifth Assessment Report of the Intergovernmental Panel on Climate Change, chap. 9, edited by: Stocker, T., Qin, D., Plattner, G.-K., Tignor, M., Allen, S., Boschung, J., Nauels, A., Xia, Y., Bex, V., and Midgley, P., Cambridge University Press, Cambridge, UK and New York, NY, USA, 741-866, 2013.

Frigessi, A., Haug, O., and Rue, H.: A dynamic mixture model for unsupervised tail estimation without threshold selection, Extremes, 5, 219-235, doi:10.1023/A:1024072610684, 2002. 
Hall, A.: Projecting regional change, Science, 346, 1461-1462, doi:10.1126/science.aaa0629, 2014.

Hasson, S., Lucarini, V., and Pascale, S.: Hydrological cycle over south and southeast Asian river basins as simulated by PCMDI/CMIP3 experiments, Earth Syst. Dynam., 4, 199-217, doi:10.5194/esd-4-199-2013, 2013.

Haylock, M. R., Hofstra, N., Klein Tank, A. M. G., Klok, E. J., Jones, P. D., and New, M.: A European daily highresolution gridded data set of surface temperature and precipitation for 1950-2006, J. Geophys. Res., 113, D20119, doi:10.1029/2008JD010201, 2008.

Hofstra, N., Haylock, M., New, M., and Jones, P. D.: Testing EOBS European high-resolution gridded data set of daily precipitation and surface temperature, J. Geophys. Res., 114, D21101, doi:10.1029/2009JD011799, 2009a.

Hofstra, N., New, M., and McSweeney, C.: The influence of interpolation and station network density on the distributions and trends of climate variables in gridded daily data, Clim. Dynam., 35, 841-858, doi:10.1007/s00382-009-0698-1, 2009b.

IPCC: Climate Change: The IPCC Scientific Assessment, Cambridge University Press, Cambridge, Great Britain, New York, NY, USA and Melbourne, Australia, 1990.

Jacob, D., Petersen, J., Eggert, B., Alias, A., Christensen, O. B., Bouwer, L. M., Braun, A., Colette, A., Déqué, M., Georgievski, G., Georgopoulou, E., Gobiet, A., Menut, L., Nikulin, G., Haensler, A., Hempelmann, N., Jones, C., Keuler, K., Kovats, S., Kröner, N., Kotlarski, S., Kriegsmann, A., Martin, E., van Meijgaard, E., Moseley, C., Pfeifer, S., Preuschmann, S., Radermacher, C., Radtke, K., Rechid, D., Rounsevell, M., Samuelsson, P., Somot, S., Soussana, J.-F., Teichmann, C., Valentini, R., Vautard, R., Weber, B., and Yiou, P.: EUROCORDEX: new high-resolution climate change projections for European impact research, Reg. Environ. Change, 14, 563-578, doi:10.1007/s10113-013-0499-2, 2013.

Katz, R.: Precipitation as a chain dependent process, J. Appl. Meteorol., 16, 671-676, doi:10.1175/15200450(1977)016<0671:PAACDP>2.0.CO;2, 1977.

Kendon, E., Roberts, N., and Fowler, H.: Heavier summer downpours with climate change revealed by weather forecast resolution model, Nat. Clim. Change, 4, 570-576, doi:10.1038/NCLIMATE2258, 2014.

Klein Tank, A. M. G., Wijngaard, J. B., Können, G. P., Böhm, R., Demarée, G., Gocheva, A., Mileta, M., Pashiardis, S., Hejkrlik, L., Kern-Hansen, C., Heino, R., Bessemoulin, P., MüllerWestermeier, G., Tzanakou, M., Szalai, S., Pálsdóttir, T., Fitzgerald, D., Rubin, S., Capaldo, M., Maugeri, M., Leitass, A., Bukantis, A., Aberfeld, R., van Engelen, A. F. V., Forland, E., Mietus, M., Coelho, F., Mares, C., Razuvaev, V., Nieplova, E., Cegnar, T., Antonio López, J., Dahlström, B., Moberg, A., Kirchhofer, W., Ceylan, A., Pachaliuk, O., Alexander, L. V., and Petrovic, P.: Daily dataset of 20th-century surface air temperature and precipitation series for the European Climate Assessment, Int. J. Climatol., 22, 1441-1453, doi:10.1002/joc.773, 2002.

Kotlarski, S., Keuler, K., Christensen, O. B., Colette, A., Déqué, M., Gobiet, A., Goergen, K., Jacob, D., Lüthi, D., van Meijgaard, E., Nikulin, G., Schär, C., Teichmann, C., Vautard, R., Warrach-Sagi, K., and Wulfmeyer, V.: Regional climate modeling on European scales: a joint standard evaluation of the EURO-
CORDEX RCM ensemble, Geosci. Model Dev., 7, 1297-1333, doi:10.5194/gmd-7-1297-2014, 2014.

Le Treut, H., Cubasch, U., and Allen, M.: Historical Overview of Climate Change Science, in: Climate Change 2007: The Physical Science Basis, Contribution of Working Group I to the Fourth Assessment Report of the Intergovernmental Panel on Climate Change, chap. 1, edited by: Solomon, S., Qin, D., Manning, M., Marquis, M., Averyt, K. B., Tignor, M., Miller, H. L., and Chen, Z., Cambridge University Press, Cambridge, UK and New York, NY, USA, 93-128, 2007.

Maraun, D.: Bias correction, quantile mapping, and downscaling: Revisiting the inflation issue, J. Climate, 26, 2137-2143, doi:10.1175/JCLI-D-12-00821.1, 2013a.

Maraun, D.: When will trends in European mean and heavy daily precipitation emerge?, Environ. Res. Lett., 8, 014004, doi:10.1088/1748-9326/8/1/014004, 2013b.

Maraun, D.: Bias Correcting Climate Change Simulations a Critical Review, Curr. Clim. Change Rep., 2, 211-220, doi:10.1007/s40641-016-0050-x, 2016.

Maraun, D. and Widmann, M.: The representation of location by a regional climate model in complex terrain, Hydrol. Earth Syst. Sci., 19, 3449-3456, doi:10.5194/hess-19-3449-2015, 2015.

Maraun, D., Wetterhall, F., Ireson, A. M., Chandler, R. E., Kendon, E. J., Widmann, M., Brienen, S., Rust, H. W., Sauter, T., Themeß1, M., Venema, V. K. C., Chun, K. P., Goodess, C. M., Jones, R. G., Onof, C., Vrac, M., and Thiele-Eich, I.: Precipitation downscaling under climate change: Recent developments to bridge the gap between dynamical models and the end user, Rev. Geophys., 48, RG3003, doi:10.1029/2009RG000314, 2010.

Maraun, D., Osborn, T. J., and Rust, H. W.: The influence of synoptic airflow on UK daily precipitation extremes. Part II: regional climate model and E-OBS data validation, Clim. Dynam., 36, 261-275, doi:10.1007/s00382-011-1176-0, 2011a.

Maraun, D., Osborn, T. J., and Rust, H. W.: The influence of synoptic airflow on UK daily precipitation extremes. Part I: Observed spatio-temporal relationships, Clim. Dynam., 36, 261275, doi:10.1007/s00382-009-0710-9, 2011b.

Maraun, D., Widmann, M., Gutiérrez, J., Kotlarski, S., Chandler, R. E., Hertig, E., Wibig, J., Huth, R., and Wilcke, R. A. I.: VALUE: A framework to validate downscaling approaches for climate change studies, Earth's Future, 3, 1-14, doi:10.1002/2014EF000259, 2015.

Meredith, E., Maraun, D., Semenov, V. A., and Park, W.: Evidence for added value of convection permitting models for studying changes in extreme precipitation, J. Geophys. Res.-Atmos., 120, 12500-12513, doi:10.1002/2015JD024238, 2015.

Michelangeli, P.-A., Vrac, M., and Loukos, H.: Probabilistic downscaling approaches: Application to wind cumulative distribution functions, Geophys. Res. Lett., 36, L11708, doi:10.1029/2009GL038401, 2009.

Palmer, T. N.: Climate extremes and the role of dynamics, P. Natl. Acad. Sci. USA, 110, 5281-5282, doi:10.1073/pnas.1303295110, 2013.

Payne, J., Wood, A., Hamlet, A., Palmer, R., and Lettenmaier, D. P.: Mitigating the effects of climate change on the water resources of the Columbia River basin, Climatic Change, 62, 233-256, doi:10.1023/B:CLIM.0000013694.18154.d6, 2004.

Petoukhov, V., Rahmstorf, S., Petri, S., and Schellnhuber, H. J.: Quasiresonant amplification of planetary waves and recent 
Northern Hemisphere weather extremes, P. Natl. Acad. Sci. USA, 110, 5336-5341, doi:10.1073/pnas.1222000110, 2013.

Piani, C., Haerter, J. O., and Coppola, E.: Statistical bias correction for daily precipitation in regional climate models over Europe, Theor. Appl. Climatol., 99, 187-192, doi:10.1007/s00704-0090134-9, 2009.

Piani, C., Weedon, G., Best, M., Gomes, S., Viterbo, P., Hagemann, S., and Haerter, J.: Statistical bias correction of global simulated daily precipitation and temperature for the application of hydrological models, J. Hydrol., 395, 199-215, doi:10.1016/j.jhydrol.2010.10.024, 2010.

Prein, A. F., Holland, G. J., Rasmussen, R. M., Done, J., Ikeda, K., Clark, M. P., and Liu, C. H.: Importance of regional climate model grid spacing for the simulation of heavy precipitation in the Colorado Headwaters, J. Climate, 26, 4848-4857, doi:10.1175/JCLI-D-12-00727.1, 2013.

Rummukainen, M.: State of the art with regional climate models, Wiley Int. Rev. Climate Change, 1, 82-96, doi:10.1002/wcc.8, 2010.

Schölzel, C. and Friederichs, P.: Multivariate non-normally distributed random variables in climate research - introduction to the copula approach, Nonlin. Processes Geophys., 15, 761-772, doi:10.5194/npg-15-761-2008, 2008.

Shao, J.: An asymptotic theory for linear model selection, Stat. Sin., 7, 221-264, 1997.

Thober, S., Mai, J., Zink, M., and Samaniego, L.: Stochastic temporal disaggregation of monthly precipitation for regional gridded data sets, Water Resour. Res., 50, 8714-8735, doi:10.1002/2014WR015930, 2014.

van der Linden, P. and Mitchell, J.: ENSEMBLES: Climate change and its impacts: Summary of research and results from the ENSEMBLES project, Tech. rep., MetOffice Hadley Centre, Exeter, UK, 2009.

van Meijgaard, E., van Ulft, L., Lenderink, G., de Roode, S., Wipfler, L., Boers, R., and Timmermans, R.: Refinement and application of a regional atmospheric model for climate scenario calculations of Western Europe, in: Climate changes Spatial Planning publication: KvR 054/12, Programme office climate changes spatial planning, Wageningen, the Netherlands, 1-45, 2012.
Volosciuk, C., Maraun, D., Semenov, V. A., and Park, W.: Extreme precipitation in an atmosphere general circulation model: Impact of horizontal and vertical model resolutions, J. Climate, 28, 1184-1205, doi:10.1175/JCLI-D-14-00337.1, 2015.

von Storch, H.: On the use of "inflation" in statistical downscaling, J. Climate, 12, 3505-3506, doi:10.1175/15200442(1999)012<3505:OTUOII>2.0.CO;2, 1999.

Vrac, M. and Naveau, P.: Stochastic downscaling of precipitation: From dry events to heavy rainfalls, Water Resour. Res., 43, W07402, doi:10.1029/2006WR005308, 2007.

Vrac, M., Drobinski, P., Merlo, A., Herrmann, M., Lavaysse, C., Li, L., and Somot, S.: Dynamical and statistical downscaling of the French Mediterranean climate: uncertainty assessment, Nat. Hazards Earth Syst. Sci., 12, 2769-2784, doi:10.5194/nhess-122769-2012, 2012.

Wong, G., Maraun, D., Vrac, M., Widmann, M., Eden, J. M., and Kent, T.: Stochastic model output statistics for bias correcting and downscaling precipitation including extremes, J. Climate, 27, 6940-6959, doi:10.1175/JCLI-D-13-00604.1, 2014.

Wood, A. W. and Maurer, E.: Long-range experimental hydrologic forecasting for the eastern United States, J. Geophys. Res., 107, 4429, doi:10.1029/2001JD000659, 2002.

Wood, A. W., Leung, L. R., Sridhar, V., and Lettenmaier, D.: Hydrologic implications of dynamical and statistical approaches to downscaling climate model outputs, Climatic Change, 62, 189 216, doi:10.1023/B:CLIM.0000013685.99609.9e, 2004.

Yee, T. W. and Stephenson, A. G.: Vector generalized linear and additive extreme value models, Extremes, 10, 1-19, doi:10.1007/s10687-007-0032-4, 2007.

Yee, T. W. and Wild, C. J.: Vector generalized additive models, J. Roy. Stat. Soc. B, 58, 481-493, 1996.

Zhang, T. and Sun, D.-Z.: ENSO Asymmetry in CMIP5 Models, J. Climate, 27, 4070-4093, doi:10.1175/JCLI-D-13-00454.1, 2014.

Zwiers, F. W., Alexander, L. V., Hegerl, G. C., Knutson, T. R., Kossin, J. P., Naveau, P., Nicholls, N., Schär, C., Seneviratne, S. I., and Zhang, X.: Climate Extremes: Challenges in estimating and understanding recent changes in the frequency and intensity of extreme climate and weather events, in: Climate Science for Serving Society, edited by: Asrar, G. R. and Hurrell, J. W., Springer Netherlands, Dordrecht, 339-389, doi:10.1007/978-94007-6692-1, 2013. 